\title{
Screening and eradication of Helicobacter pylori for gastric cancer prevention: the Taipei global consensus
}

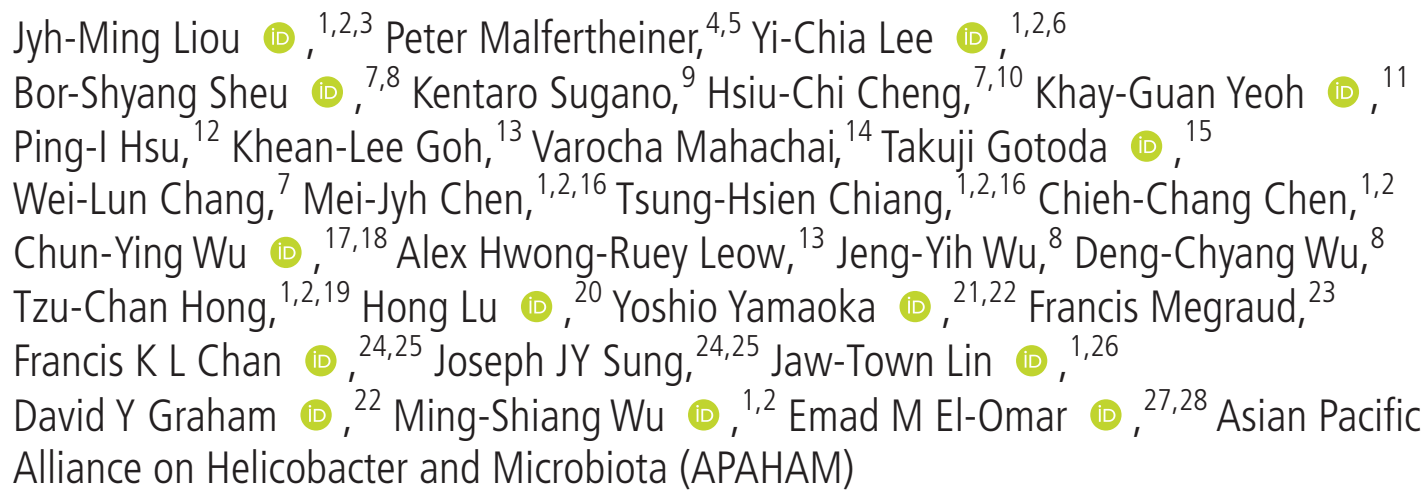

- Additional material is published online only. To view, please visit the journal online (http://dx.doi.org/10.1136/ gutjnl-2020-322368).

For numbered affiliations see end of article.

\section{Correspondence to}

Professor Ming-Shiang Wu, Department of Internal Medicine, National Taiwan University Hospital, Taipei 10002, Taiwan;

mingshiang@ntu.edu.tw

J-ML and PM contributed equally.

DYG, M-SW and EME-O are joint senior authors.

Received 28 June 2020 Revised 27 July 2020 Accepted 12 August 2020

Published Online First

1 October 2020

\section{Check for updates}

(C) Author(s) (or their employer(s)) 2020. No commercial re-use. See rights and permissions. Published by BMJ.

To cite: Liou J-M

Malfertheiner $\mathrm{P}$, Lee $\mathrm{Y}-\mathrm{C}$, et al. Gut 2020;69:2093-2112.

\section{ABSTRACT}

Objective A global consensus meeting was held to review current evidence and knowledge gaps and propose collaborative studies on population-wide screening and eradication of Helicobacter pylori for prevention of gastric cancer (GC).

Methods 28 experts from 11 countries reviewed the evidence and modified the statements using the Delphi method, with consensus level predefined as $\geq 80 \%$ of agreement on each statement. The Grading of Recommendation Assessment, Development and Evaluation (GRADE) approach was followed.

Results Consensus was reached in 26 statements. At an individual level, eradication of $H$. pylori reduces the risk of GC in asymptomatic subjects and is recommended unless there are competing considerations. In cohorts of vulnerable subjects (eg, first-degree relatives of patients with $\mathrm{GC}$ ), a screen-and-treat strategy is also beneficial. $H$. pylori eradication in patients with early GC after curative endoscopic resection reduces the risk of metachronous cancer and calls for a re-examination on the hypothesis of 'the point of no return'. At the general population level, the strategy of screen-and-treat for $\mathrm{H}$. pylori infection is most cost-effective in young adults in regions with a high incidence of $\mathrm{GC}$ and is recommended preferably before the development of atrophic gastritis and intestinal metaplasia. However, such a strategy may still be effective in people aged over 50 , and may be integrated or included into national healthcare priorities, such as colorectal cancer screening programmes, to optimise the resources. Reliable locally effective regimens based on the principles of antibiotic stewardship are recommended. Subjects at higher risk of GC, such as those with advanced gastric atrophy or intestinal metaplasia, should receive surveillance endoscopy after eradication of $H$. pylori.

Conclusion Evidence supports the proposal that eradication therapy should be offered to all individuals infected with H. pylori. Vulnerable subjects should be tested, and treated if the test is positive. Mass screening and eradication of $\mathrm{H}$. pylori should be considered in populations at higher risk of GC.

\section{INTRODUCTION}

Despite a recent decline in incidence, gastric cancer remains one of the leading causes of cancer death worldwide. ${ }^{1}$ The major breakthrough in research on gastric cancer occurred with the discovery of Helicobacter pylori and proof that the infection was aetiologically related to gastric cancer. The organism has been classified as a class 1 human carcinogen. ${ }^{2}$ H. pylori causes a pattern of gastritis described as acute-on-chronic inflammation, which leads to chronic progressive gastric damage and ultimately to gastric atrophy. Gastric cancer is an inflammationassociated malignancy in which the infection directly and indirectly causes progressive genetic damage to the gastric epithelium that may eventually lead to gastric adenocarcinoma. Risk of developing gastric cancer in $H$. pylori infected individuals can be estimated based on the degree and extent of mucosal damage and atrophy, recognised as metaplastic epithelia with or without intraepithelial neoplasia or dysplasia. Interventional trials and studies in humans have shown that eradication of $H$. pylori can reduce the risk of gastric cancer, which is related to the extent of genetic alterations and epigenetic modifications present at the time of $H$. pylori eradication. ${ }^{3-7}$ However, there is a great gap in translating this basic and clinical knowledge into public health intervention through population-wide screening and eradication of $H$. pylori, which ultimately prevent the development of gastric cancer. First, we lack updated data on the global disease burden of $H$. pylori infection and gastric cancer and we need to better identify the target populations (general population or selected vulnerable populations) for such screening and eradication programmes. Second, the implementation of mass screening requires consideration of the choice of non-invasive test, when to proceed to endoscopy, whether and how to test for eradication efficacy and how to survey those with advanced atrophic gastritis, how to implement this programme, how to treat asymptomatic $H$. pylori infected subjects for gastric cancer prevention, and how to identify subjects at higher risk of gastric cancer for endoscopic 
surveillance after H. pylori eradication. Additionally, there are also some concerns about the widespread use of antibiotics, including the emergence of antibiotic resistance of bacteria other than $H$. pylori, the perturbation of gut microbiota, potential effects on the risk of extragastric disorders, such as obesity, gastro-oesophageal reflux disease, metabolic syndrome, and autoimmune diseases. ${ }^{89} \mathrm{In}$ December 2013, the International Agency for Research on Cancer (IARC) organised a working group meeting to review the evidence for eradication of $H$. pylori as a strategy for prevention of gastric cancer, and published a working group report. ${ }^{10}{ }^{11}$ In September 2019, we held a monothematic scientific conference (10th Asian Pacific topic conference) organised by the Asian Pacific Association of Gastroenterology (APAGE), the Japanese Society of Gastroenterology (JSGE) and the Gastroenterological Society of Taiwan (GEST) under the theme of "screening and eradication of $H$. pylori infection for gastric cancer prevention". After the topic conference, we held an expert consensus meeting in Taipei to review current evidence, identify knowledge gaps and propose collaborative studies to resolve these concerns on mass screening and eradication of $H$. pylori for gastric cancer prevention.

\section{METHODS}

\section{Consensus development process}

Five major topics were chosen by core members of the organising committee (J-ML, Y-CL, B-SS, EME-O and M-SW). Drafts of clinical questions (CQs) about each topic were prepared by J-ML and were further revised by core members (PM, Y-CL, DYG, EME-O and M-SW). Altogether, 33 CQs were selected for the first round of voting. Faculty members selected from members of APAGE, JSGE, GEST, European Helicobacter and Microbiota Study Group and Healthy Stomach Initiative were assigned to one or two of the five subgroups according to their expertise. The selection of core members was based on expertise. The selection of faculty members was based on both expertise and geographical representativeness. The faculty members (J-ML, Y-CL, B-SS, H-CC, KG-Y, W-LC, M-JC, T-HC, C-CC, C-YW, P-IH and M-SW) were assigned to perform systematic reviews of CQs 1-3 and to prepare the statements, which were edited in the first steering committee on 20 July 2019. The revised draft statements were further edited by moderators and core members (J-ML, PM, Y-CL, B-SS, DYG, EME-O and M-SW). The Delphi method was used for consensus development. The Grading of Recommendation Assessment, Development and Evaluation (GRADE) system was applied for grading of recommendation and evidence level (online supplementary table $1 \mathrm{~S}$ and $2 \mathrm{~S}) .{ }^{12}$ Consensus level was predefined as $\geq 80 \%$ of agreement on each statement.

The supporting evidence for each statement were revised by core members (J-ML, PM, Y-CL, B-SS, DYG, EME-O and M-SW) and was then emailed to all faculty members 2 weeks before the face-to-face meeting in Taipei. The first voting was done by all faculty members via the electronic voting system 1 week before the meeting. They were asked to indicate their agreement (agree or not agree), levels of recommendation (strong, weak, weak against or strong against), level of evidence, and reasons for disagreement for each statement. The statements were further revised by the core members (J-ML, PM, FM, EME-O and $\mathrm{M}-\mathrm{SW}$ ) accordingly. The supporting evidence for each clinical question was presented in the plenary lecture during the topic conference on 28 and 29 September 2019 in Kaohsiung. The face-to-face consensus meeting was held on 30 September and 1 October 2019 in Taipei. The statements and level of evidence were discussed and revised by all faculty members in a plenary session and were then uploaded to the Zuvio electronic voting pad system for the second round of anonymous voting. The level of agreement and grade of recommendation for each statement were shown on the screen in real time. Statements which failed to reach $\geq 80 \%$ of agreement during the second round of voting were further discussed and modified and voting carried out again, if necessary. Seven statements which failed to reach consensus of $\geq 80 \%$ despite this process were discarded. A total of 26 statements passed this process and were voted on in the third round. Three statements (Nos 8, 10 and 18) which failed to reach $\geq 80 \%$ of agreement in the third round voting were further discussed and modified. Consensus was then reached in the fourth round voting for these three statements.

\section{Role of the funding sources}

The consensus meeting was funded by GEST with support from industries, National Taiwan University Hospital, and Ministry of Science and Technology of Taiwan. The funding sources had no role in the planning and organisation of the meeting, study design, data collection, analysis or interpretation, report writing or the decision to submit this manuscript for publication. All authors had full access to the data and participated in the decision to submit for publication.

\section{RESULTS}

The statements that reached consensus are summarised in table 1.

\section{DISEASE BURDEN OF H. PYLORI INFECTION ASSOCIATED GASTRIC CANCER}

\section{CQ 1. Is gastric cancer still a public health threat and} underestimated in the world?

Statement 1: Although the global age-standardised incidence and mortality rate of gastric cancer is decreasing, the number of new cases of gastric cancer remains high due to an increase of the elderly population.

Agreement: agree (96\%).

Grade of recommendation: strong (84\%), weak (16\%), weak against (0\%), strong against (0\%).

Evidence level: moderate.

\section{Comments}

Gastric cancer remains the fifth most common cancer and the third leading cause of cancer deaths worldwide. ${ }^{1}$ The 5 -year survival rate of advanced gastric cancer is lower than 30\% in Europe. ${ }^{1}$ The estimated age-standardised incidence in 2018 was 15.7 and 7.0 per 100000 in males and in females worldwide, respectively according to the GLOBOCAN 2018 database (figure 1A). ${ }^{13}$ The estimated age-standardised incidence was as high as 30.2 and 12.5 per 100000 in males and in females in the WHO Western Pacific region, respectively (figure $1 \mathrm{~A}) .{ }^{1}$ The estimated age-standardised incidence was highest in Korea, Mongolia, Japan, China and Kyrgyzstan (figure 1B). ${ }^{13}$ There were more than one million new cases worldwide in 2018 and more than 60\% (640 000) of them occurred in the WHO Western Pacific region (figure 1C). The estimated new cases were highest in China, Japan, India and Korea. ${ }^{1}$ The estimated lifetime (up to age 74 years) cumulative risk of incidence of gastric cancer was $1.9 \%$ and $0.8 \%$ in males and females worldwide, respectively, and was highest in the WHO Western Pacific region (figure 1D). It is estimated that gastric cancer develops in 6.9\%, 5.6\%, 4.9\% and 3.5\% of males in Korea, Mongolia, Japan and China, respectively (figure 1E). ${ }^{113}$ Although it is expected that the incidence of gastric cancer will be further reduced in the next two decades, the number of new cases is expected to increase because of the increase of the elderly 
Table 1 Summary of consensus recommendations for screening and eradication of $H$. pylori for gastric cancer prevention

\begin{tabular}{|c|c|c|c|c|}
\hline No. & Statement & Agree & Evidence level & Limitations of current evidence and areas for future research \\
\hline \multicolumn{5}{|c|}{ Disease burden of $H$. pylori infection-associated gastric cancer } \\
\hline 1 & $\begin{array}{l}\text { Although the global age-standardised incidence and mortality rate of } \\
\text { gastric cancer is decreasing, the number of new cases of gastric cancer } \\
\text { remains high due to an increase of the elderly population }\end{array}$ & $96 \%$ & Moderate & $\begin{array}{l}\text { Lack of cancer registration database in many countries or regions; } \\
\text { updated prevalence of } H \text {. pylori needed for more accurate estimation of } \\
\text { future disease burden }\end{array}$ \\
\hline $2-1$. & $\begin{array}{l}\text { Although the prevalence of } \mathrm{H} \text {. pylori is decreasing in most Western } \\
\text { countries, it remains high in populations with a high incidence of gastric } \\
\text { cancer }\end{array}$ & $96 \%$ & Low & $\begin{array}{l}\text { Lack of updated prevalence in many countries; potential selection bias, } \\
\text { age-standardised prevalence not reported and accuracy of test not } \\
\text { validated in some studies }\end{array}$ \\
\hline $2-2$. & $\begin{array}{l}\text { The prevalence of } H \text {. pylori in children has fallen below } 10 \% \text { in some } \\
\text { populations, but remains high in many parts of the world }\end{array}$ & $96 \%$ & Low & $\begin{array}{l}\text { Lack of updated prevalence in many countries and potential selection } \\
\text { bias }\end{array}$ \\
\hline 3. & $\begin{array}{l}\text { The worldwide attributable fraction for } H \text {. pylori in gastric cancer }(\mathrm{GC}) \text { is } \\
\text { higher than } 85 \% \text {, indicating that the majority of GC can be prevented if } \\
\text { H. pylori infection is eliminated from a population }\end{array}$ & $88 \%$ & Moderate & $\begin{array}{l}\text { Estimation based on nested case-control studies in Western } \\
\text { populations }\end{array}$ \\
\hline 4. & $\begin{array}{l}\text { Eradication of } H \text {. pylori reduces the risk of gastric cancer in infected } \\
\text { subjects }\end{array}$ & $92 \%$ & Moderate & $\begin{array}{l}\text { Trials conducted in Eastern populations, except one from Columbia, } \\
\text { number of cases of gastric cancer relatively small; progression of } \\
\text { gastric precancerous lesions as primary outcome in several trials, risk } \\
\text { reduction for intestinal type and diffuse type not known, long-term } \\
\text { adverse consequences not assessed, eradication rate } \sim 70 \% \text {, reinfection } \\
\text { rate } \sim 2-7 \% \text { /year }\end{array}$ \\
\hline
\end{tabular}

5. Eradication of $H$. pylori after resection of early gastric cancer is $\quad 96 \% \quad$ High $\quad$ Nearly all were intestinal type gastric cancer recommended because it reduces the risk of metachronous gastric cancer

Implementation of $H$. pylori screening and eradication programme at population level

6. Screening and eradication of $H$. pylori for gastric cancer prevention is $84 \% \quad$ Low recommended in populations with a high incidence or high risk of gastric cancer

7. Screening and eradication of $H$. pylori before the development of atrophic $84 \% \quad$ Low gastritis and intestinal metaplasia is recommended

8. The strategy of screen-and-treat for $H$. pylori infection is most cost$84 \% \quad$ Low effective in young adults for gastric cancer prevention in regions with a high incidence of gastric cancer

9. Young individuals would benefit most from H. pylori eradication because $92 \%$ it cures $H$. pylori related gastritis, reduces the risk of gastric cancer and reduces transmission to their children

10. A urea breath test or $\mathrm{H}$. pylori stool antigen test are the preferred tests for mass screening, but a locally validated serology test may be considered

11. In H. pylori infected individuals, endoscopy is additionally recommended $100 \%$ for those with a higher risk for gastric cancer

12. Population-wide screening and eradication of $H$. pylori infection should be integrated or included in national healthcare priorities to optimise the resources

Treatment of $H$. pylori infection in mass eradication programmes

Estimation derived from cost-effectiveness analysis, lack of direct evidence from randomised trial, prevalence of $H$. pylori should also be considered

Lack of direct evidence from randomised trials, the age of development of precancerous lesions varies according to gender and ethnicity Assumption based on observational studies rather than randomised trials, saving related to dyspepsia or peptic ulcer disease rarely considered in the models, benefit reported by life-years saved rather than QALYS

Low Lack of randomised trials showing the reduction of gastric cancer risk in young individuals and the transmission

Lack of direct comparison of the accuracy and acceptability of three non-invasive tests in mass screening

Low

Prospective studies needed for risk stratification in populations with different incidence of gastric cancer

Population-wide screening and eradication programme only in Japan and some regions in China, Korea and Taiwan

13. There is a trend of increasing resistance rates to clarithromycin and levofloxacin worldwide

14. The antibiotic resistance profile of $H$. pylori in different regions, efficacy, adverse effects and cost should be taken into account in choosing the optimal regimens in the community

15 Reliable locally effective regimens based on the principles of antibiotic stewardship are recommended

16. Surveillance of the local antibiotic resistance of $H$. pylori is recommended $96 \%$ to identify the optimal empirical therapy for mass eradication of $H$. pylori in that population

17. The reinfection rate after $H$. pylori eradication is very low

radication of $H$. pylori does not increase the risk of new onset GORD

Confirmation test of $H$. pylori eradication is not mandatory in mass screening, but should be performed in subsets of the population for assessment of treatment efficacy

Potentially adverse consequences of $H$. pylori eradication

19. As with all antibiotic treatments, H. pylori eradication may lead to an increase in antimicrobial resistance, but it should not preclude its use for gastric cancer prevention

20. Short-term perturbation of faecal microbiota diversity occurs after $\mathrm{H}$. pylori eradication, which largely recovers subsequently GORD

22. H. pylori eradication may be associated with a small increase in body weight, but does not increase the risk of metabolic syndrome

$\begin{array}{ll}96 \% & \text { Moderate } \\ 96 \% & \text { Low }\end{array}$

$100 \% \quad$ Low

$100 \% \quad$ Low

$92 \%$

Moderate

Moderate

Low

$92 \% \quad$ Very low

$88 \%$

Low

$92 \%$

$96 \%$

Treatment-experienced subjects not excluded in some, different breakpoint of MICs used, lack of updated data in many countries Priority of efficacy, adverse effects and cost in community settings remains debatable

The impact of following the antibiotic stewardship principle needs to be assessed in the community

Resistance rate might vary in different regions in the same country and may change with time

Few studies reported the reinfection rate in the mass screening and eradication in the community

Formal cost-effectiveness analysis using data from prospective trials is needed to assess the necessity of confirmation test in all subjects

Scarce evidence regarding the long-term impacts of eradication therapy on the antimicrobial resistance at individual and population levels

Scarce evidence regarding the long-term impacts of eradication therapy on the composition of human microbiota, especially at species level

$80 \% \quad$ Low

Well-designed randomised trials are needed to assess the impacts of eradication therapy on human metabolism and metabolic disorders 


\begin{tabular}{|c|c|c|c|c|}
\hline No. & Statement & Agree & Evidence level & Limitations of current evidence and areas for future research \\
\hline 23. & $\begin{array}{l}\text { H. pylori eradication does not increase the risk of asthma, inflammatory } \\
\text { bowel disease and other immune-related diseases }\end{array}$ & $80 \%$ & Very low & $\begin{array}{l}\text { Lack of evidence from randomised trials or large-scale prospective } \\
\text { cohort studies }\end{array}$ \\
\hline \multicolumn{5}{|c|}{ Endoscopic surveillance for gastric cancer after $H$. pylori eradication } \\
\hline 24. & $\begin{array}{l}\text { Subjects with advanced gastric atrophy or intestinal metaplasia should } \\
\text { receive surveillance endoscopy to detect gastric cancer after } H \text {. pylori } \\
\text { eradication }\end{array}$ & $92 \%$ & Low & $\begin{array}{l}\text { Evidence from retrospective studies with relatively small sample size. } \\
\text { Eradication not confirmed in some studies }\end{array}$ \\
\hline 25. & $\begin{array}{l}\text { Surveillance endoscopy is suggested every } 2 \text { to } 3 \text { years for subjects with } \\
\text { advanced gastric atrophy or intestinal metaplasia, and every } 12 \text { months } \\
\text { after the removal of neoplasia }\end{array}$ & $92 \%$ & Low & $\begin{array}{l}\text { Large-scale prospective cohort studies/randomised trials/cost- } \\
\text { effectiveness analysis are warranted to assess the optimal surveillance } \\
\text { interval }\end{array}$ \\
\hline 26. & $\begin{array}{l}\text { Genetic and epigenetic markers show promise in stratifying gastric } \\
\text { cancer risk after } H \text {. pylori eradication, but require further validation in } \\
\text { prospective studies }\end{array}$ & $92 \%$ & Low & $\begin{array}{l}\text { Studies are needed to assess the role of serum markers, endoscopic } \\
\text { features, histological grading and molecular markers in risk } \\
\text { stratification }\end{array}$ \\
\hline
\end{tabular}

GORD, gastro-oesophageal reflux disease; MICs, minimum inhibitory concentrations; QALYs, quality of life years.

population (figure 1F). However, cancer registries are not available in many countries. The incidence of gastric cancer is probably underestimated in countries with limited medical and endoscopic resources. On the other hand, the prevalence of $H$. pylori infection is decreasing dramatically in other countries. Updated data are needed to produce a more accurate estimate of the current and future disease burden of gastric cancer.

\section{CQ 2. Does $\boldsymbol{H}$. pylori infection, the major cause of gastric cancer, remain prevalent in the world?}

Statement 2-1: Although the prevalence of $H$. pylori is decreasing in most Western countries, it remains high in populations with a high incidence of gastric cancer.

Agreement: agree (96\%).

Grade of recommendation: strong (76\%), weak (24\%), weak against (0\%), strong against (0\%).

Evidence level: low.

Statement 2-2: The prevalence of $H$. pylori in children has fallen below $10 \%$ in some populations, but it remains high in many parts of the world.

Agreement: agree (96\%).

Grade of recommendation: strong (68\%), weak (28\%), weak against (0\%), strong against (4\%).

Evidence level: low.

\section{Comments}

A systematic review and meta-analysis showed that the prevalence of $H$. pylori infection was highest in Africa (79.1\%), Latin America and the Caribbean (63.4\%), and Asia (54.7\%), but was lower in Northern America (37.1\%) and Oceania (24.4\%) in the general adult population from 1970 through $2016 .{ }^{14}$ It was estimated that 4.4 billion of people are infected with $H$. pylori worldwide. ${ }^{14}$ However, the prevalence of $H$. pylori is decreasing in the younger generations ( $<40$ years) in many populations. Recent studies showed significant reduction in the prevalence of $H$. pylori in several countries, including the United States, Japan and Taiwan. ${ }^{15-17}$ The updated prevalence of $H$. pylori during 20152020 in the world is shown in figure $2 \mathrm{~A}$ and online supplementary table $3 \mathrm{~S}$.

The majority of $H$. pylori infections are acquired in childhood, usually before the age of $10 .^{18}$ Therefore, the prevalence of $H$. pylori infection in children may reflect the disease burden in that population decades later. Recent studies showed that the prevalence of $H$. pylori in children has been fallen below 10\% in some populations, such as Germany, Japan, Korea, Taiwan and Hong Kong. ${ }^{16}{ }^{19-21}$ In contrast, the prevalence of $H$. pylori is still higher than $10 \%$ in many paediatric populations, such as Latin America,
Middle East and Africa. ${ }^{20}$ The prevalence of $H$. pylori in children and adolescents in studies published during 2010-2020 is shown in figure $2 \mathrm{~B}$.

However, there are several limitations to the current evidence concerning the global prevalence of $H$. pylori infection. First, updated data on the prevalence are limited, making it difficult to estimate the true level of $H$. pylori worldwide. Second, the tests used to survey the prevalence of $H$. pylori were heterogeneous and the accuracy of these tests varied. Third, the methods used to recruit study subjects and the inclusion and exclusion criteria also varied. Fourth, the age-standardised prevalence of $H$. pylori infection was not reported in the majority of these studies. Since the prevalence of $H$. pylori is usually higher in older people and lower in the younger generations, studies that recruit a higher proportion of older people may overestimate the actual disease burden of $H$. pylori infection. Therefore, it is crucial to survey the updated prevalence of $H$. pylori using the same study protocol and validated tests. It is suggested that the age-standardised prevalence of that population and the prevalence according to birth years should be reported in future studies.

\section{CQ 3. What is the proportion of gastric cancer attributable to H. pylori infection?}

Statement 3: The worldwide attributable fraction for H. pylori in gastric cancer is higher than $85 \%$, indicating that the majority of gastric cancer can be prevented if $H$. pylori infection is eliminated from a population.

Agreement: agree (88\%).

Grade of recommendation: strong (68\%), weak (28\%), weak against $(0.0 \%)$, strong against (4\%).

Evidence level: moderate.

\section{Comments}

The aetiology of gastric cancer (adenocarcinoma) is heterogeneous. Hereditary diffuse-type gastric cancer accounts for 1-2\% of gastric cancer and is attributable to germline mutations in certain genes, such as the E-cadherin gene which is associated with a penetrance rate of greater than $60 \% .^{22}$ Adenocarcinoma of the cardia accounts for $10-15 \%$ of gastric cancer and is mostly associated with gastrooesophageal reflux disease, obesity and cigarette smoking. ${ }^{23}$ The aetiology of cancer at the gastro-oesophageal junction is heterogeneous, and it can arise in advanced atrophic gastric mucosa attributable to $H$. pylori if two-thirds of the tumour extends into the stomach. ${ }^{24}$ Epstein-Barr virus associated with lymphoepitheiomalike carcinoma accounts for $4-6 \%$ of gastric cancer. ${ }^{25}$ Interactions of $H$. pylori infection with dietary habits (eg, high salt diet), cigarette smoking and host genetic factors contribute to the development 
(A)

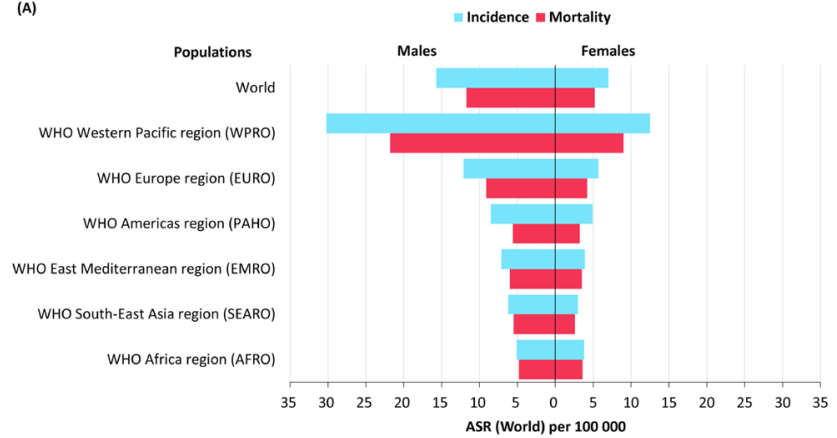

(c)

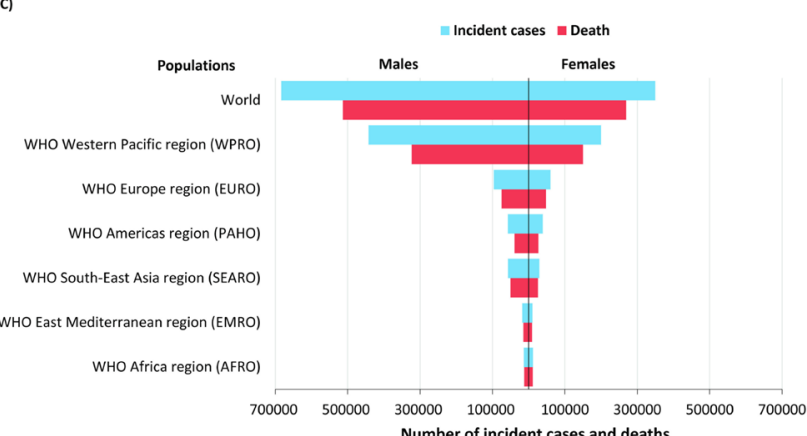

(E)

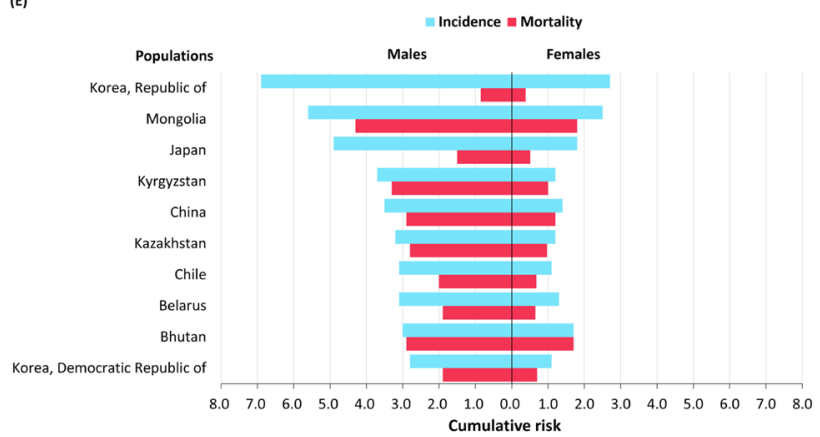

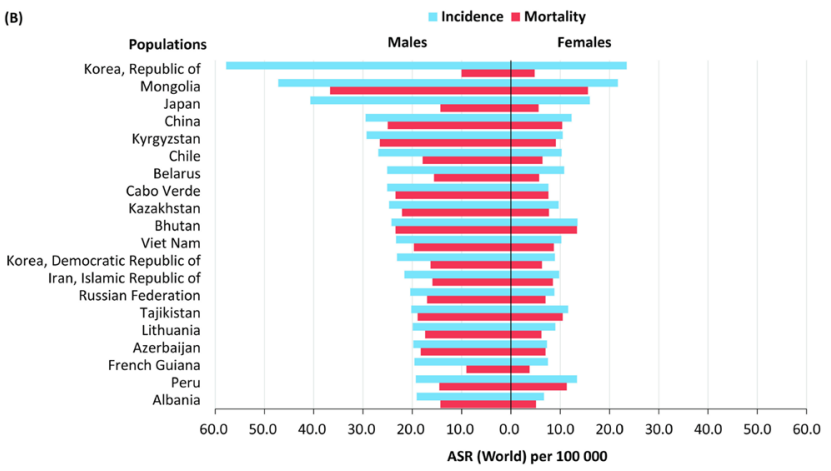

(D)

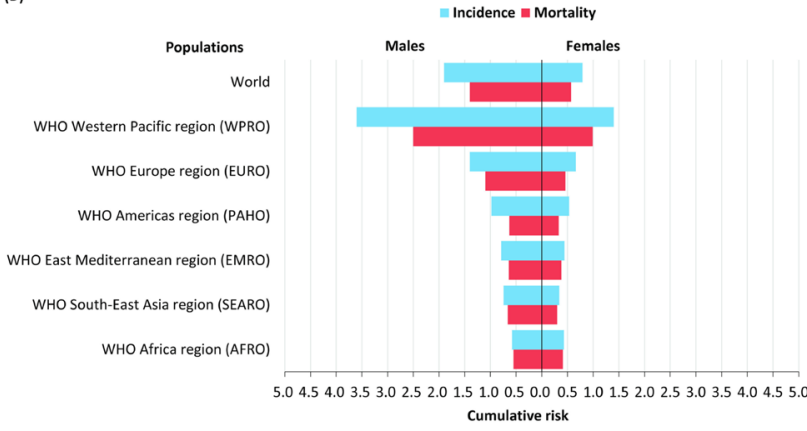

(F)

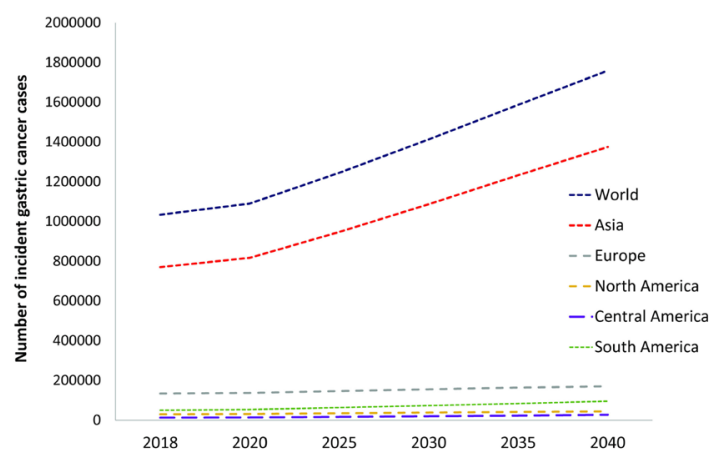

Figure 1 Estimated disease burden of gastric cancer in 2018 according to GLOBOCAN database. ${ }^{13}$ (A) Incidence and mortality of gastric cancer according to WHO regions. ${ }^{13}$ (B) Countries with highest incidence of gastric cancer. ${ }^{13}$ (C) Number of patients with gastric cancer and death according to WHO regions. ${ }^{13}$ (D) Cumulative risk of incidence and mortality of gastric cancer according to WHO regions. ${ }^{13}$ (E) Countries with highest cumulative risk of incidence of gastric cancer in 2018, ages $0-74 .{ }^{13}$ (F) Predicted number of incident cases of gastric cancer in the next two decades. ${ }^{13}$ ASR, agestandardised incidence.

of non-cardia gastric cancer. ${ }^{9}$ Cohort studies in Japan and Taiwan showed that $1-2 \%$ of $H$. pylori infected subjects developed gastric cancer. ${ }^{26}$ Interestingly, they showed that none of the H. pylori uninfected subjects developed gastric cancer after a median follow-up of 8-10 years. ${ }^{26}$ Using both enzyme-linked immunosorbent assay and multiple antigen immunoblot for detection of $H$. pylori infection in four nested case-control studies, Plummer et al estimated that the worldwide attributable fraction of $H$. pylori for non-cardia gastric cancer is $89 \%$, indicating that more than $85 \%$ of non-cardia gastric cancer could be prevented if $H$. pylori were not present in that population. ${ }^{27}$

\section{CQ 4. Does H. pylori eradication reduce the risk of gastric cancer in $\mathbf{H}$. pylori infected subjects?}

Statement 4: Eradication of $H$. pylori reduces the risk of gastric cancer in infected subjects.
Agreement: agree (92\%).

Grade of recommendation: strong (92\%), weak (4\%), weak against (0\%), strong against (4\%).

Evidence level: moderate.

\section{CQ 5. Does $\boldsymbol{H}$. pylori eradication reduce the risk of} metachronous gastric cancer after curative endoscopic resection of early gastric cancer?

Statement 5: Eradication of $H$. pylori after resection of early gastric cancer is recommended because it reduces the risk of metachronous gastric cancer.

Agreement: agree (96\%).

Grade of recommendation: strong (88\%), weak (8\%), weak against $(0 \%)$, strong against $(4 \%)$.

Evidence level: high. 
(A)

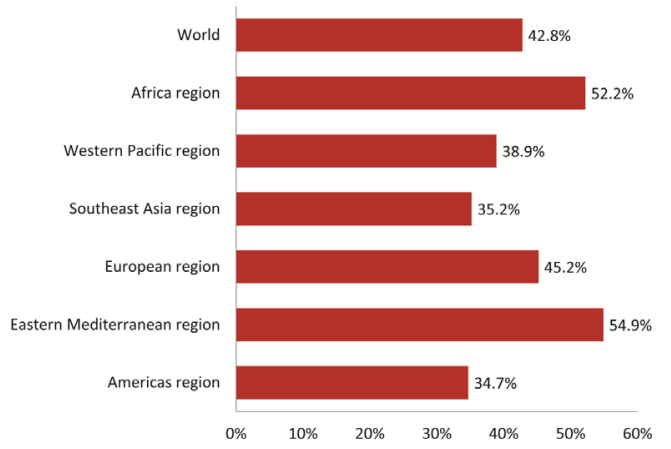

(B)

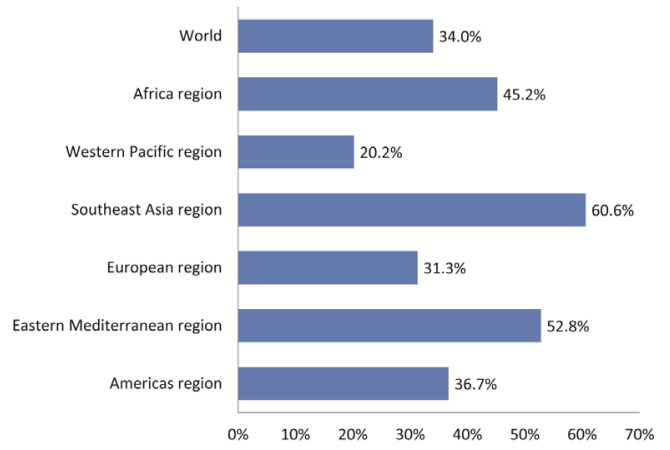

Figure 2 Updated global prevalence of $H$. pylori infection (reference to online supplementary table 3). (A) Prevalence of $H$. pylori in adults (2015-2020). (B) Prevalence of $H$. pylori in children (2010-2020).

\section{Comments}

Seven randomised clinical trials including a total of 8834 healthy $H$. pylori infected subjects receiving eradication therapy $(n=4461)$ versus no treatment or placebo $(n=4373)$ for primary prevention of gastric cancer have been reported (table 2). ${ }^{3528-35}$ The mean age was about 50 years at baseline and about half of them were males. The eradication rates at baseline ranged from $70 \%$ to $84 \%$. At the end of follow-up
(4-22 years), gastric cancer developed in 71 and 127 subjects among the treated and non-treated groups, respectively (risk ratio (RR) $0.55,95 \% \mathrm{CI} 0.42$ to 0.74 ). It is noteworthy that eradication of $H$. pylori appears to be ineffective for the prevention of gastric cancer in the two trials that included $100 \%$ of subjects with precancerous lesions, including lowto high-grade dysplasia at baseline. ${ }^{28} 35$ In subgroup analysis, Wong et al showed that eradication therapy was significantly better than placebo in subjects without precancerous lesions at baseline. ${ }^{3}$ This indicates that eradication of $H$. pylori before the development of precancerous lesions offers better protection against gastric cancer. A community-based study conducted in the Matsu Islands, Taiwan, showed a short-term reduction of $25 \%$ in the incidence of gastric cancer around 5 years after mass eradication. After long-term follow-up of 12 years, a significant reduction of $53 \%$ was observed and a greater reduction of $68 \%$ would be expected by $2025 .^{36} 37$

There are 3 randomised controlled trials included a total of 1841 patients with gastric cancer who received curative endoscopic resection and eradication $(n=910)$ versus no treatment or placebo $(n=931)$ for secondary prevention of gastric cancer (table 2). ${ }^{4839}$ The mean age was greater than 60 years at baseline and more than $70 \%$ of them were male. The eradication rates ranged from $75 \%$ to $83 \%$. At the end of follow-up (3-6 years), gastric cancer developed in 40 and 87 patients in the treated and non-treated groups, respectively (RR 0.48 , 95\% CI 0.33 to 0.69 ). Meta-analysis of another 10 prospective or retrospective cohort studies also showed that eradication therapy may reduce the risk of metachronous gastric cancer after curative endoscopic resection (RR $0.44,95 \%$ CI 0.33 to $0.58) .^{40}$ The result indicates that eradication therapy is still effective for gastric cancer prevention in older patients with gastric cancer who already have precancerous lesions in their stomach.

The effectiveness of eradication in healthy $H$. pylori infected subjects for gastric cancer prevention is being assessed in another two large-scale double blind randomised trials in China and Korea (table 3). ${ }^{41}$ The effectiveness of $H$. pylori eradication has also been assessed in another randomised controlled trial in the UK, in which the primary outcome is peptic ulcer bleeding. Three ongoing trials in UK, Taiwan, and Latvia are examining whether screening and eradication of $H$. pylori is effective in reducing the incidence of gastric cancer (table 3 ).

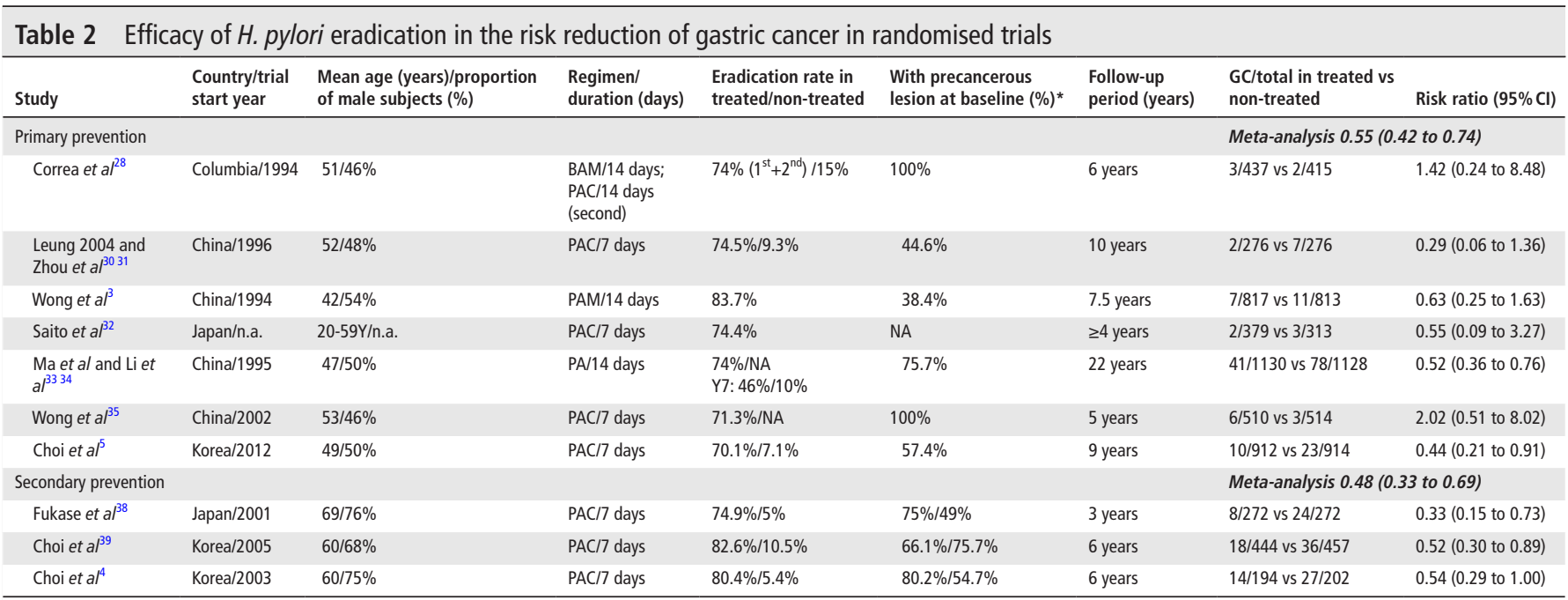

*the extent and severity of precancerous lesions varied among studies.

BAM, bismuth, amoxicillin and metronidazole; GC, gastric cancer; NA, not available; PAC, proton pump inhibitor, amoxicillin, clarithromycin; 1st+2nd, overall eradication rates. 


\section{IMPLEMENTATION OF H. PYLORI SCREENING AND ERADICATION PROGRAMME AT POPULATION LEVEL CQ 6. Who should we actively screen and treat $\boldsymbol{H}$. pylori?}

Statement 6: Screening and eradication of $H$. pylori for gastric cancer prevention is recommended in populations with a high incidence or high risk of gastric cancer.

Agreement: agree (84\%).

Grade of recommendation: strong (64\%), weak (32\%), weak against (0\%), strong against (4\%).

Evidence level: low.

\section{Comments}

There are two levels of questions about the effectiveness of screening and eradication of $H$. pylori for gastric cancer prevention. First, can eradication therapy reduce the risk of gastric cancer in those with $H$. pylori infection (figure 3A)? Second, can screening and eradication of $H$. pylori reduce the risk of gastric cancer in the general population (figure 3B). Ten randomised trials showed that eradication of $H$. pylori can reduce the risk of gastric cancer in asymptomatic infected subjects. H. pylori may still be effective in those receiving curative endoscopic resection for early gastric cancer, as shown in statements 4 and 5 . The effectiveness of $H$. pylori eradication for gastric cancer prevention is essentially dependent on the efficacy of the treatment regimen and patients' adherence to it. The accuracy of the test for $H$. pylori infection, to follow the eradication success and the reinfection/recrudescence rate is critical (figure 3A). Only one randomised trial has reported the effectiveness of screening and eradication of $H$. pylori for gastric cancer prevention, but three clinical trials are ongoing that will provide information in the next few years (table 3 ). The effectiveness of such strategy is expected to be higher if the participation rate for $H$. pylori screening ( 1$)$, the prevalence of $H$. pylori infection in that population (p2), the true positive rate of the H. pylori test (p3), the participation rate for eradication therapy $(\mathrm{p} 4)$, the eradication rate of treatment $(\mathrm{p} 5)$ and the sustained eradication rate (p6) are higher. A high false-negative rate of the $H$. pylori testing (p7) may reduce effectiveness, whereas a high false-positive rate may increase the number of patients receiving unnecessary antibiotics (figure 3B). Additionally, the efficacy of eradication therapy to reduce gastric cancer can also be influenced by the rate of mortality/incidence rate, which is an indirect estimate of early detection. ${ }^{42}$

The magnitude of the protective effect of $H$. pylori eradication also depends on the baseline risk. The relation of the baseline risk and the magnitude of the protective effect from $H$. pylori eradication is reported in a meta-analysis that included 24 studies, in which the benefit was most evident in populations with an incidence rate higher than 150 per 100000 person-years. ${ }^{6}$ This observation is a reflection of the natural history of gastric cancer, which is characterised by a long silent (latent) period during which atrophic gastritis/gastric atrophy is developing. When a sufficient proportion of the population has developed atrophy, the risk switches from linear to exponential (ie, from 150 to 300 to 600 per 100000 person-years, etc). ${ }^{6}$ Thus, eradication in the linear phase will largely prevent gastric cancer altogether, whereas cure after a sizeable proportion has developed atrophy and a definite risk will have a more dramatic effect but be overall less effective for that population. ${ }^{6}$ Randomised trials have shown that $H$. pylori eradication can reduce the risk of metachronous gastric cancer in patients who have received endoscopic resection of early gastric cancer by up to $50 \% .^{48-40}$ For all populations, active testing and treatment for $H$. pylori infection will reduce (or prevent) gastric cancer. ${ }^{43}$ The numerical benefits are greatest in high-risk populations and in these test-and-treat should be routinely performed. It is important to note that even intermediate- or low-risk populations can contain subpopulations or immigrants with higher gastric cancer risk and are candidates for active screening. It is also important to assess whether the test-and-treat strategy could reduce the mortality rate of gastric cancer, which requires a longer follow-up period than decline of the incidence rate.

On the individual level, ethnicity, immigration history, family history and lifestyle habits, prior endoscopic histories and the positive results of non-invasive testing for $H$. pylori and related damage to the gastric mucosa (eg, pepsinogen testing) should be used to identify high-risk subjects who should receive endoscopic/histological assessment first or receive immediate treatment for $H$. pylori infection. ${ }^{44}$

The risk and incidence of gastric cancer vary greatly according to ethnicity, gender and geographical regions. Countries or populations with an incidence of gastric cancer greater than 20 per 100000 person-years, between 10 and 20 per 100000 personyears, and lower than 10 per 100000 person-years are defined as high-, intermediate- and low-risk populations according to prior consensus meetings. The 20 countries with highest incidence of gastric cancer shown in figure $1 \mathrm{~B}$ are categorised as high-risk population accordingly. The annual incidence was greater than 100 per 100000 person-years in men aged 55 or older in Korea and Japan, and in men aged 65 years or older in China and Brazil. Additionally, certain populations from a low-incidence country may have a higher risk of gastric cancer, such as indigenous populations in Alaska, USA.

Table 3 The efficacy of $H$. pylori eradication or screen-and-eradication strategy in the risk reduction of gastric cancer: ongoing trials

\begin{tabular}{|c|c|c|c|c|c|c|c|}
\hline Country registration number & Subjects/design & Experiment group & Control group & Primary outcome & Age (years) & Sample size/status & $\begin{array}{l}\text { Start year/expected } \\
\text { completion year }\end{array}$ \\
\hline \multicolumn{8}{|l|}{ Effectiveness of $H$. pylori eradication } \\
\hline China/ChiCTR-TRC-10000979 & Healthy subjects/double blind & $\begin{array}{l}\text { Bismuth quadruple } \\
\text { therapy }\end{array}$ & $\begin{array}{l}\text { Bismuth + omeprazole } \\
+ \text { placebo }\end{array}$ & Gastric cancer incidence & $25-54$ & $\begin{array}{l}94101 / \text { recruitment } \\
\text { completed }\end{array}$ & 2011-2013/NA \\
\hline Korea/NCT02112214 & $\begin{array}{l}\text { Healthy H. pylori infected } \\
\text { subjects/double blind }\end{array}$ & $\begin{array}{l}\text { Bismuth quadruple } \\
\text { therapy }\end{array}$ & Placebo & Gastric cancer incidence & $40-60$ & $\begin{array}{l}\text { 5224/recruitment } \\
\text { completed }\end{array}$ & $2014 / 2029$ \\
\hline UK/NCT01506986 & $\begin{array}{l}\text { H. pylori-infected aspirin user/ } \\
\text { double blind }\end{array}$ & Triple therapy & Placebo & Peptic ulcer bleeding & $\geq 60$ & $\begin{array}{l}\text { 3038/recruitment } \\
\text { completed }\end{array}$ & $2012 / 2021$ \\
\hline \multicolumn{8}{|c|}{ Effectiveness of screening and eradication of $H$. pylori } \\
\hline UK/ISRCTN71557037 & Healthy subjects/open label & H. pylori screening & No screening & $\begin{array}{l}\text { Gastric cancer incidence and } \\
\text { mortality }\end{array}$ & $\begin{array}{l}\text { M: 35-69; } \\
\text { F:45-69 }\end{array}$ & $\begin{array}{l}56000 / \text { recruitment } \\
\text { completed }\end{array}$ & $1997 / 2021$ \\
\hline Taiwan/NCT01741363 & Healthy subjects/open label & $\begin{array}{l}\text { H. pylori screening } \\
\text { and FIT }\end{array}$ & FIT alone & Gastric cancer incidence & $50-69$ & $\begin{array}{l}60000 / \text { recruitment } \\
\text { completed }\end{array}$ & $2014 / 2017$ \\
\hline Latvia/NCT02047994 & Healthy subjects/open label & $\begin{array}{l}\text { H. pylori screening } \\
\text { and FIT }\end{array}$ & FIT alone & Gastric cancer mortality & $40-64$ & 30 000/recruiting & $2013 / 2033$ \\
\hline
\end{tabular}

$\mathrm{FIT}$, faecal immunochemical test; NA, not available. 

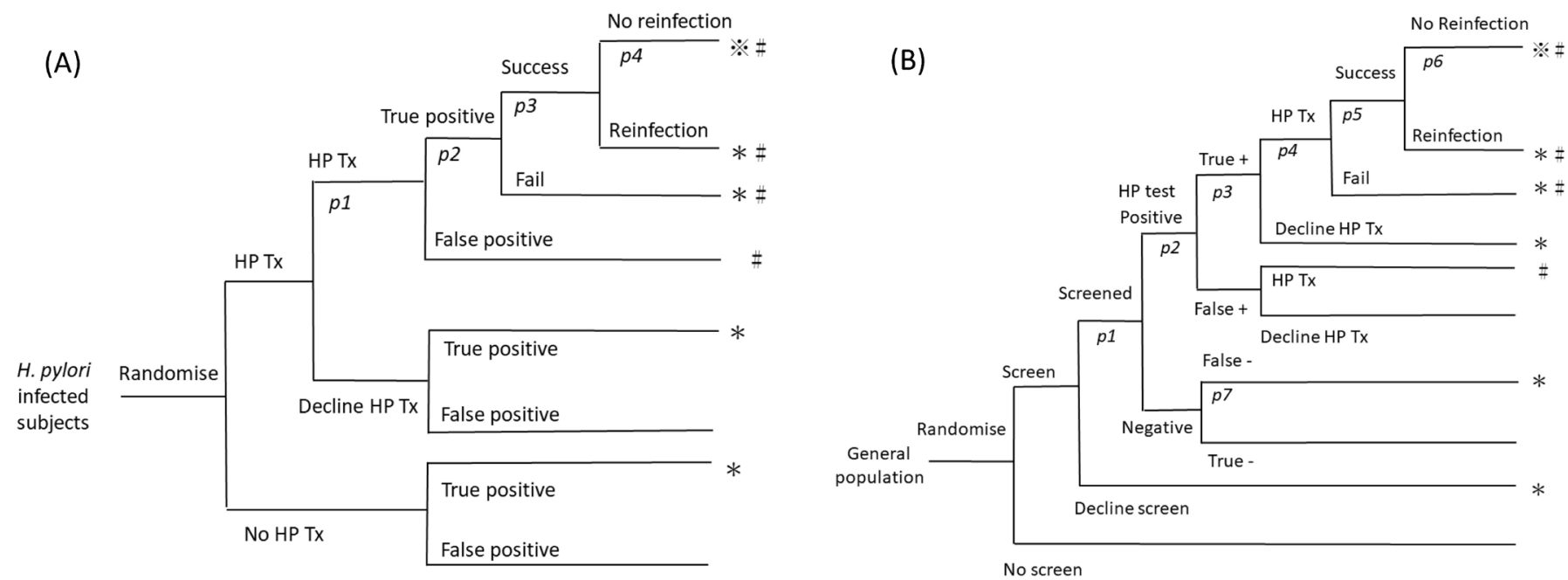

(C)
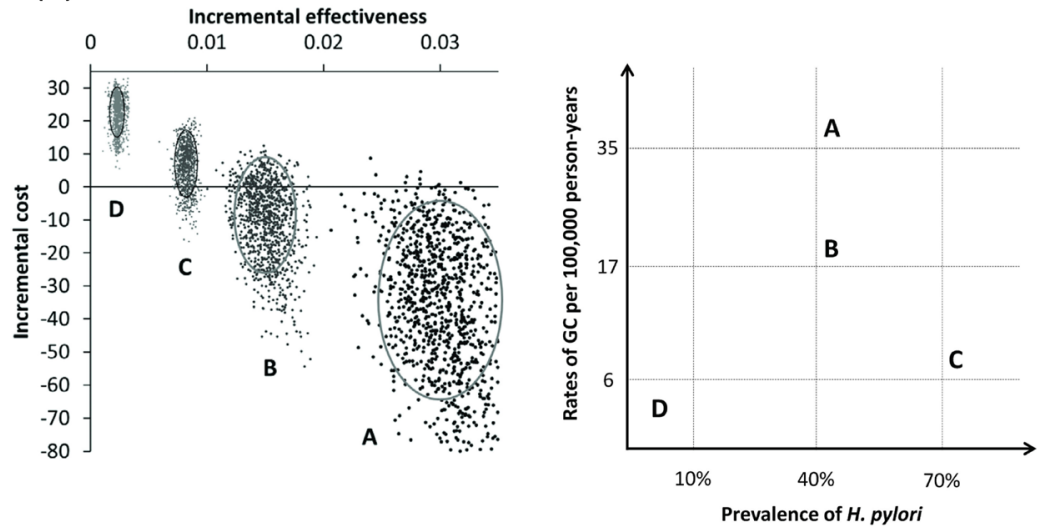

Figure 3 Effectivenss and cost-effectiveness of screening and eradication of $H$. pylori for prevention of gastric cancer. (A) Effectiveness of eradication therapy for $H$. pylori infected subjects. (B) Effectiveness of screening and eradication of $H$. pylori: ※may benefit most from screen-andtreat (risk reduction of gastric cancer); * partial or no benefit from screen-and-treat; \#potential adverse consequences from eradication therapy. (C) Cost-effectiveness of mass eradication for young individuals according to different rates of gastric cancer and $H$. pylori infection. The analyses are based on a previously developed Markov decision model (Matsu Islands, Taiwan) by modifying the risk of gastric cancer and the rate of $H$. pylori infection. ${ }^{52}$ With the starting age of 20 years, the model makes a comparison of the relative cost-effectiveness between 1000 individuals with or without the intervention. The results are presented on the cost-effective plane (US dollars per life-year gained); the elliptical circles indicate the $95 \%$ confidence intervals. Four scenarios are demonstrated: (A) both high rates of gastric cancer (35 per 100000 person-years) and H. pylori (40\%); (B) an intermediate rate of gastric cancer (17 per 100000 person-years) and a high rate of H. pylori (40\%); (C) a low rate of gastric cancer (6 per 100000 person-years) but a high rate of $H$. pylori (70\%); and (D) low rates of both gastric cancer (6 per 100000 person-years) and $H$. pylori infection (10\%). The results indicate that for high- and intermediate-risk populations, screening for young individuals is associated with higher effectiveness but a lower cost (ie, cost saving) as compared with no screening. For the low-risk populations, screening is associated with the higher effectiveness and the higher cost, so the choices depend on how much society is willing to pay for a life-year gained.

\section{CQ 7. When should we actively screen and treat $H$. pylori?}

Statement 7: We recommend screening and eradication of $H$. pylori before the development of atrophic gastritis and intestinal metaplasia.

Agreement: agree (84\%).

Grade of recommendation: strong (68\%), weak (28\%), weak against (0\%), strong against (4\%).

Evidence level: low.

\section{Comments}

It is estimated that more than $85 \%$ of non-cardia gastric cancer is attributable to $H$. pylori infection. ${ }^{27}$ However, a pooled analysis based on real-world data from eradication trials demonstrated only a $46 \%$ risk reduction in subjects who received $H$. pylori treatment as compared with those did not. ${ }^{67} \mathrm{~A}$ retrospective cohort study based on the national health insurance database showed early $H$. pylori eradication may reduce the risk of gastric cancer by $23 \%$ compared with those who did not receive timely treatment. ${ }^{45}$ The discrepancy in the magnitude of benefit between the expected and observed levels probably reflects the proportion of patients whose chronic gastritis has progressed to a stage with preneoplastic changes and genetic alterations so they maintained a cancer risk despite getting rid of the bacteria.

The increase in gastric cancer risk and the underlying increase in genetic instability follow a recognisable histological pattern, starting from chronic active gastritis to progressive atrophic gastritis with development of metaplastic epithelia. Intraepithelial neoplasia then appears and finally invasive carcinoma (ie, an updated version of the original Correa cascade) ${ }^{46} \mathrm{H}$. pylori eradication stops the progression of injury but is unlikely to entirely reverse the genetic instability that has occurred. Therefore, screening and eradication of $H$. pylori is ideally implemented before the development of irreversible genetic instability, which is usually reflected histologically in the development of atrophic 
gastritis and metaplasia epithelia. ${ }^{43}$ Age could be an indicator of gastric precancerous lesion, but this varies according to ethnicity. Atrophic gastritis appears at a younger age in North East Asia but occurs after the age of 50-55 years in Western populations. ${ }^{47}$ Yet, a retrospective cohort study using the Hospital Authority database of Hong Kong showed that eradication of $\mathrm{H}$. pylori may also reduce the risk of gastric cancer in subjects older than 60 years. $^{48}$

\section{CQ 8. Is screening and eradication of $\boldsymbol{H}$. pylori cost-effective for gastric cancer prevention?}

Statement 8: The strategy of screen-and-treat for H. pylori infection is most cost-effective in young adults for gastric cancer prevention in regions with a high incidence of gastric cancer.

Agreement: agree (84\%).

Grade of recommendation: strong (68\%), weak (28\%), weak against (0\%), strong against (4\%).

Evidence level: low.

\section{Comments}

Systematic reviews showed that the screen-and-treat strategy for H. pylori is cost-effective for gastric cancer prevention. ${ }^{49} 50$ Five variables determine cost-effectiveness in the sensitivity analysis. ${ }^{49}$ The first is the prevalence of $H$. pylori. The cost per lifeyear saved (LYS) of the strategy ranges from US\$ 10000 to 35000 in the low-prevalence regions and US\$ 200 to 17000 in high-prevalence regions, such as Columbia and Singapore. ${ }^{50}$ The second is the estimated proportion of gastric cancer reduced. If the cancer reduction rate is $\geq 15 \%$, the incremental costeffectiveness ratio (ICER) would be less than US\$ 50000 per LYS, the most common threshold value. ${ }^{51}$ The third variable is age at screening, which depends on the population screened. In Western countries, a study showed that screening at ages $>50$ has low ICER values, which increase if the screening starting age drops. ${ }^{49}$ However, in Eastern Asian people, it is more costeffective when the screening starting age is at 20 to 30 years rather than at an older age. ${ }^{52-54}$ Moreover, the strategy may be cost-effective only in Japanese-Americans after age $10 .^{51}$ The fourth is the incidence of gastric cancer. In specific high-risk groups, such as those with early gastric cancer after endoscopic resection, ICER is less than US\$ 4000 per LYS. ${ }^{55}$ The fifth variable is the cost of tests or cancer treatment. ${ }^{49}{ }^{50}$ Limitations of current evidence include the lack of assumptions based on results from randomised control trials, savings related to dyspepsia or peptic ulcer diseases were rarely considered in the models, and benefit reported as life-years saved in the elderly rather than as quality-adjusted life-years (QALYs).

\section{CQ 9. Who will benefit most from the $H$. pylori treatment for gastric cancer prevention?}

Statement 9: Young individuals would benefit most from $H$. pylori eradication because it cures $H$. pylori related gastritis, reduces the risk of gastric cancer and reduces transmission to their children.

Agreement: agree (92\%).

Grade of recommendation: strong (56\%), weak (40\%), weak against (4\%), strong against (0\%).

Evidence level: low.

\section{Comments}

H. pylori infection is usually acquired during childhood so that the patient's age reflects the duration of the infection and, within a specific population, is an indicator of the extent of the inflammation-associated damage. ${ }^{6}$ Even though evidence from comparative studies is limited, simulation studies have repeatedly indicated that screening at a younger age (20-40 years) is more cost-effective because the efficacy of $H$. pylori eradication for averting gastric cancer is greatest at that age. ${ }^{52-54}$ In addition to the reduction of cancer risk, screening of younger individuals has additional benefits. First, the risk of other $H$. pylorirelated diseases can be reduced, such as peptic ulcer disease, non-ulcer dyspepsia, iron deficiency anaemia, etc, so the overall cost-effectiveness is augmented. ${ }^{54}$ Second, curing the infection before young adults begin their families can reduce the risk of intrafamilial transmission. A number of studies have reported that the presence of an $H$. pylori infected mother is associated with a higher risk of transmitting $H$. pylori to their children (OR of 13). ${ }^{56}$ Thus, screening young adults, but not children or adolescents, is likely to be an effective approach to preventing transmission to children and protect those who are not infected within the family.

\section{CQ 10. Which test should we use for mass screening of $H$. pylori infection?}

Statement 10: Urea breath test or $H$. pylori stool antigen test are the preferred tests for mass screening, but a locally validated serology test may be considered.

Agreement: agree (88\%).

Grade of recommendation: strong (63\%), weak (33\%), weak against (4\%), strong against $(0 \%)$.

Evidence level: moderate.

\section{Comments}

Non-invasive tests, including the ${ }^{13} \mathrm{C}$-urea breath test $\left({ }^{13} \mathrm{C}\right.$ UBT), H. pylori stool antigen (HpSA) test ${ }^{57}$ and serology test $\mathrm{t}^{58}$ are available for mass screening of $H$. pylori in the community. Direct comparison of the performance of these three tests in mass screening is lacking. In a Cochrane review of hospital-based studies, indirect comparison showed that ${ }^{13} \mathrm{C}$-UBT appears to be more accurate than serology (diagnostic odds ratio 3.2, 95\% CI 1.2 to 8.4 ) and HpSA test (diagnostic odds ratio 3.4, 95\% CI 1.3 to 8.8$).{ }^{59}$ However, the diagnostic accuracy was comparable if the assessment was restricted to seven head-to-head studies. ${ }^{59}$ Factors that might influence the cost-effectiveness for mass screening include the prevalence of $H$. pylori infection, patient adherence, cost of the test and gastric cancer treatment, additional benefit of the testing, incidence of gastric cancer and estimated cancer reduction. ${ }^{49}{ }^{60}{ }^{13} \mathrm{C}$-UBT is accurate but is more expensive. The HpSA test is as accurate but is less expensive than ${ }^{13} \mathrm{C}$-UBT. However, the acceptability of the HpSA test for mass screening might be lower, and delayed delivery of stool samples might cause degradation of the antigens and, in turn, false-negative results. Serology testing is the least expensive and convenient but does not distinguish between active and past infection. A model showed that ${ }^{13} \mathrm{C}$-UBT is more cost-effective than HpsA if the prevalence of $H$. pylori is higher than $25 \%$, but HpSA is more cost-effective if the adherence rate is higher than $63 \%$. $^{49}$ In a mass screening programme for subjects aged 50-69 years in Changhua County, Taiwan, a two-in-one approach was used to detect faecal occult blood for colorectal cancer screening and $H$. pylori antigen for gastric cancer prevention simultaneously, thus increasing the beneficial effect of mass screening. ${ }^{61}$ Locally validated serology tests with high sensitivity may also be acceptable for mass screening, but it is suggested that eradication therapy should be offered after confirmation by ${ }^{13} \mathrm{C}$-UBT or the HpSA test. 
CQ 11. In addition to non-invasive screening for $H$. pylori, who may benefit from endoscopy for the detection of asymptomatic gastric cancer?

Statement 11: In $H$. pylori infected individuals, endoscopy is additionally recommended for those with a higher risk for gastric cancer.

Agreement: agree (100\%).

Grade of recommendation: strong (72\%), weak (28\%), weak against $(0 \%)$, strong against $(0 \%)$.

Evidence level: low.

\section{Comments}

Some asymptomatic subjects might already have gastric cancer or precancerous gastric lesions in their stomach at the time of screening. Therefore, endoscopy should be provided for subjects at higher risk of gastric cancer, including populations with high incidence and individuals with higher risk factors for gastric cancer, such as older age, male gender, family history of gastric cancer in first-degree relatives, serum pepsinogen I/II ratio $<3$ or cigarette smokers. ${ }^{47}{ }^{62-67}$ In most high-risk populations, a starting age around 50 years has been generally recommended based on the notion that the endoscopic yield rate for the premalignant gastric lesion and gastric cancer is likely to have increased to a level that is considered cost-effective. ${ }^{4466} 67$ The risk of gastric cancer is higher in males and therefore the cut-off age for endoscopy should be different in males and females. For residents from low-incidence areas, the diagnostic yield based on the criterion of age is not practical and the magnitude of gastric cancer risk must be individualised. For example, family history can be considered a valuable indicator; research has shown a 2.4-fold increased risk for subjects who had a positive family history compared with those who did not. ${ }^{66}$ Reduction of gastric cancer achieved by $H$. pylori eradication in the vulnerable group of first-degree relatives is as high as $73 \% .^{5}$ Serological testing based on levels of pepsinogen I/II levels and ratio can also identify the risk of atrophic gastritis, ${ }^{44}{ }^{67}$ which then requires confirmation by endoscopy with histology.

\section{CQ 12. How to implement the screen-and-treat strategy for $\boldsymbol{H}$. pylori at the population level?}

Statement 12: Population-wide screening and eradication of $H$. pylori infection should be integrated or included in the national healthcare priorities to optimise the resources.

Agreement: agree (92\%).

Grade of recommendation: strong (64\%), weak (32\%), weak against $(0 \%)$, strong against (0\%).

Evidence level: low.

\section{Comments}

To adopt this strategy as a healthcare policy, one must consider multiple steps, including the invitation, participation, testing and referral, before the eradication treatment. The service should be delivered by following the principle of an organised screening programme. ${ }^{68}$ Priorities must be set by considering which patients to screen, when is the best time to screen, whether this strategy is cost-effective and how to increase the diagnostic yield, in order to integrate the policy into national healthcare systems.

To realise population-wide screening and eradication of $H$. pylori, one needs to consider whether the presence of other diseases will compete with the limited resources or whether screening services can be integrated into the established framework to reduce the cost. ${ }^{68}$ In high-risk populations, such as countries with an annual incidence of gastric cancer greater than
20 people/100,000, implementation of a screen-and-treat policy is straightforward because it can be supported by the higher population motivation and better cost-effectiveness. As shown in figure 3C, the relative cost-effectiveness of mass eradication is defined according to the risk of gastric cancer and prevalence of H. pylori. For example in Taiwan, since 2004, mass screening and treating $H$. pylori infection has been implemented in a high-risk population of the Matsu Islands, and this has been accompanied by a rapid decline in the incidence of gastric cancer and the risk of peptic ulcer disease. ${ }^{36}$ In Japan, starting 2013, such a national policy has been included as one of the healthcare priorities when people are receiving an endoscopic examination; an accelerating decline of gastric cancer incidence is observed.$^{69}$ Both policies are supported by the higher effectiveness at the lowest cost in reducing the burden of $H$. pylori related disease. In intermediaterisk populations, other diseases are more likely to compete for the limited resources, so the policy making should consider more how to optimise the resources-for example, incorporation of the screening service within the existing healthcare framework can substantially reduce the cost. $^{70}$ Even in low-risk populations, such an approach is still applicable as there are probably some high-risk ethnicities or immigrants from high-risk areas, ${ }^{71}$ for whom, active screening can reduce the cancer risk and also reduce the risk of $H$. pylori transmission in the community. ${ }^{72}$

\section{TREATMENT OF H. PYLORI INFECTION IN MASS ERADICATION PROGRAMMES}

\section{CQ 13. Is the antibiotic resistance rate of $H$. pylori increasing} worldwide?

Statement 13: There is a trend of increasing resistance rates to clarithromycin and levofloxacin worldwide.

Agreement: agree (100\%).

Grade of recommendation: strong (76\%), weak (24\%), weak against $(0 \%)$, strong against $(0 \%)$.

Evidence level: low.

\section{Comments}

A systematic review and meta-analysis showed that the overall prevalence of primary $H$ pylori resistance in Asia-Pacific regions was $17 \%(95 \%$ CI $15 \%$ to $18 \%)$ for clarithromycin, $44 \%(95 \%$ CI $39 \%$ to $48 \%$ ) for metronidazole, $18 \%(95 \%$ CI $15 \%$ to $22 \%)$ for levofloxacin, $3 \%(95 \%$ CI $2 \%$ to $5 \%)$ for amoxicillin and $4 \%$ (95\% CI $2 \%$ to 5\%) for tetracycline between 1990, and $2016 .^{73}$ They further showed a significant increase of clarithromycin $(21 \%)$ and levofloxacin resistance $(27 \%)$ in this region during 2011-2015, compared with those reported before 2000, whereas resistance to amoxicillin, tetracycline and metronidazole remained stable. ${ }^{73}$ A similar trend is observed globally. ${ }^{74}$ A comprehensive review of the global prevalence of resistance showed that the primary and secondary resistance rates to clarithromycin and levofloxacin were $\geq 15 \%$ in the majority of WHO regions. ${ }^{74}$ However, there are some limitations of the current evidence. First, data from numerous countries are lacking, especially the updated prevalence of resistance after 2016. Second, some studies included strains from treated patients, which may overestimate the actual primary resistance rate. Third, the methods and the break points of minimum inhibitory concentrations used to determine the antibiotic resistance varied in different studies. Fourth, the study periods of the published articles varied greatly. Finally, the samples were obtained from a single centre and the sample sizes were small in several studies. Therefore, surveillance of the updated prevalence of primary antibiotic resistance of $H$. pylori is warranted. 
CQ 14. Are there special considerations in choosing the optimal regimens for mass eradication of $H$. pylori in the community?

Statement 14: The antibiotic resistance profile of $H$. pylori in different regions, efficacy, adverse effects and cost should be taken into account in choosing the optimal regimens in the community.

Agreement: agree (100\%).

Grade of recommendation: strong (68\%), weak (32\%), weak against $(0 \%)$, strong against (0\%).

Evidence level: low.

\section{CQ 15. Should we modify the treatment of $\boldsymbol{H}$. pylori mass eradication for gastric cancer prevention?}

Statement 15: Reliable locally effective regimens based on the principles of antibiotic stewardship are recommended.

Agreement: agree (92\%).

Grade of recommendation: strong (76\%), weak (20\%), weak against $(0 \%)$, strong against (4\%).

Evidence level: moderate.

\section{CQ 16. Should susceptibility testing guided therapy be adopted or is empirical therapy according to the local prevalence of antibiotic resistance preferred for mass $\mathrm{H}$. pylori eradication?}

Statement 16: Surveillance of the local antibiotic resistance of $H$. pylori is recommended to identify the optimal empirical therapy for mass eradication of $H$. pylori in that population.

Agreement: agree (96\%).

Grade of recommendation: strong (84\%), weak (16\%), weak against $(0 \%)$, strong against $(0 \%)$.

Evidence level: moderate.

\section{Comments}

These recommendations are based on the recent recognition that $H$. pylori should be considered and treated as any other common bacterial infection by applying the principles of antimicrobial stewardship. This change represents a radically different approach from treatment than has been used since the discovery of $H$. pylori and requires much of the available data to be re-examined and some discarded. Antimicrobial stewardship is a multifaceted approach that requires considering treatment policies, guidelines, surveillance of regional emerging resistance and prevalence reports of antibiotic resistance. Furthermore, antimicrobial stewardship demands continuous education and audits of practice by healthcare organisations to optimise prescribing. ${ }^{75}$ It also includes coordinated interventions designed to improve and measure appropriate use of antimicrobial agents by promoting selection of the optimal drug regimen, including dosing, duration of treatment and route of administration. ${ }^{75-77}$

\section{Effective therapy}

Subjects to be considered when selecting a regimen for treatment of an infectious disease include effectiveness, simplicity, tolerability, adverse effects, the prevalence of antibiotic resistance in the community, dose, duration and costs. In the specific condition of gastric infection, optimal control of gastric $\mathrm{pH}$ is needed, because this has a fundamental influence on the antibiotic bioavailability in the stomach. ${ }^{24} 447778$ The requirement for excellent adherence emphasises the need for patient education and for use of regimens with problems of adherence only when necessary. Only locally highly reliably regimens should be used empirically and, if used, treatment success should be monitored such that use of the preferred regimen can be discontinued if resistance begins to undermine its effectiveness. Highly successful regimens can also be used empirically if treatment success is monitored so that their use can be stopped if resistance begins to undermine their effectiveness.

\section{Antimicrobials and proton pump inhibitors}

As shown in box 1, a wide range of antimicrobial agents and antimicrobial doses have been used successfully. The optimum dosing adopted in a certain region (area) or for the individual patient must depend on both the treatment regimen and the host (eg, CYP2C19). In the presence of actual or suspected metronidazole resistance, higher doses of metronidazole and longer duration are typically best (ie, $1600-2000 \mathrm{mg}$ of metronidazole given four times a day for 14 days). The optimum proton pump inhibitors (PPIs)/potassium-competitive acid blockers (P-CABs) and dosing regimen may vary, depending on the population (eg, drug metabolism, degree of corpus gastritis) and the composition of the regimen (ie, variably influenced by gastric acidity), and will need to be considered for its effect on acid suppression rather than the names or number of milligrams of the drugs used. ${ }^{77-79}$

\section{Box 1 Components of effective $H$. pylori therapies}

\section{Components and dosing frequency}

Antimicrobial agents

- Amoxicillin: 500-3000 mg/day

- Bismuth: most use 300-600 mg /two to four times a day

- Clarithromycin (macrolides): 400-1000 mg/day

- Metronidazole/tinidazole: $800-1000 \mathrm{mg} /$ day in triple therapy

- Metronidazole/tinidazole: 1600-2000 mg/day in bismuth quadruple therapy

- Fluoroquinolones (levofloxacin): $500 \mathrm{mg} /$ day

- Tetracycline HCl: $500 \mathrm{mg}$ four times a day

- Rifabutin: $150-300 \mathrm{mg} /$ day

- Furazolidone: $100 \mathrm{mg}$ three times a day

- Nitazoxanide: 500 mg twice a day

Antisecretory drugs

- Proton pump inhibitors: $20 \mathrm{mg}$ (or $40 \mathrm{mg}^{\star}$ ) omeprazole or equivalent dose* two times a day.

- Potassium competitive acid blockers: vonoprazan $20 \mathrm{mg}$ b.i.d.

Not recommended

- Doxycycline

- Nitazoxanide

- Probiotics

Elements of antimicrobial stewardship

- Optimise prescribing

- Promote responsible antibiotic use

- Ensure sustainable access to effective treatment (ie, prevent development of resistance)

Problems with effective anti-H. pylori therapy

- Inoculum effect (preexistence of a population of resistant strains)

- Persister effect (presence of non-replicating or slowly replicating bacterial population that requires the duration of treatment to be prolonged)

* The use of higher dosage and the definition of equivalent dose remain controversial. 


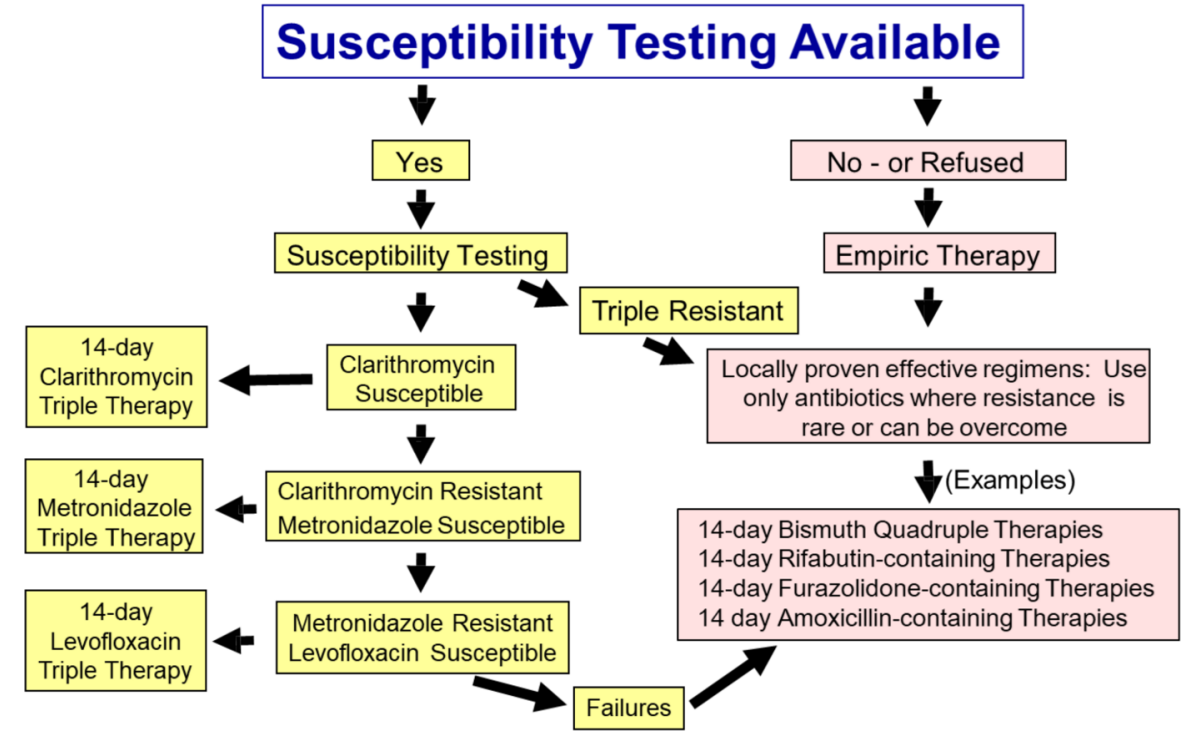

Figure 4 An example of decision-making for the appropriate use of antibiotics for $H$. pylori eradication.

\section{Duration of treatment}

The recent guidelines have recommended treatment duration of 14 days unless a shorter period is locally proved to be noninferior and reliably produces a suitably high success rate. ${ }^{24} 6280$ The optimum duration of treatment is based on overcoming the persister effect and takes into account the fact that PPIs do not achieve full effectiveness in acid suppression until after 3 or 4 days of administration. ${ }^{78}$ P-CABs, however, achieve full effectiveness on day 1 of administration and provide higher and more consistent $\mathrm{pH}$ control. This suggests that shorter durations may be equally effective. ${ }^{79}{ }^{81} 82$ Future studies are warranted to assess the efficacy of high-dose dual therapy containing P-CABs and the optimum duration of treatments with P-CABs.

\section{Susceptibility-guided therapy versus empirical therapy}

Ideally all antimicrobial therapies are susceptibility-based. ${ }^{83}$ By definition, this includes that both regimens are designed to take into account patient- and population-specific susceptibility results as well as highly successful empirically derived regimens, in which susceptibility is identified by trial and error rather than by direct testing. Antimicrobial stewardship includes the goal of ensuring sustainable access to effective therapies, which means that the regimen must not promote antimicrobial resistancethat is, higher than necessary doses must not be used, durations should not be longer than necessary, and especially, antibiotics that cannot benefit the patient (unnecessary antibiotics) should not be given. Ideally, the best results can be achieved by a stepwise approach using the most effective, best tolerated regimen first and the most complicated last (figure 4). Such an approach has been tested in highly resistant populations in China and has proved to be simple and highly effective. ${ }^{84}$

However, cost and convenience dictate that a proven reliable highly effective empiric regimen would generally be preferred initially, if such a regimen is available. In practice, antimicrobial therapy for most infections relies on a regimen proved to be highly effective locally with the expectation that ongoing antimicrobial surveillance programmes or test of cure in the majority of patients will inform when it is necessary to change and will also provide appropriate recommendations (ie, antimicrobial stewardship in action). In patients for whom empirical therapy fails in a mass eradication programme, susceptibility testing guided therapy or an alternative proven highly reliable empiric therapy is suggested.

Treatment failures occur and provide feedback to the clinician about the effectiveness of their current regimens, which when shared, provides information to the community about current regimens. All consensus groups recommend routine testing for cure to ensure treatment success. This information should also be collected and shared to inform when previously effective regimens are beginning to lose effectiveness.

\section{CQ 17. Is the reinfection rate high after mass eradication of H. pylori?}

Statement 17: The reinfection rate after mass $H$. pylori eradication is very low.

Agreement: agree (96\%).

Grade of recommendation: strong (72\%), weak (28\%), weak against $(0 \%)$, strong against $(0 \%)$.

Evidence level: moderate.

\section{Comments}

Recurrence after confirmed eradication of $H$. pylori infection can occur either by recrudescence or reinfection. Recrudescence is defined as reappearance of the original strain which is undetected by the false negative confirmatory test. Reinfection is defined as infection by a new strain after successful eradication therapy. A previous study showed that of the 10 strains obtained from patients who experienced recurrence during the first year, four strains were genetically different from the initial strain, although this could be related to the dominance of a recrudescent strain. ${ }^{85} 86$ The other six were identical to the initial strains. ${ }^{85}$ However, all the four strains obtained from patients who experienced recurrent infections after the second year were different from the initial strains. ${ }^{85}$ Factors that might contribute to recrudescence include therapeutic regimen, shorter length of treatment, confirmatory test provided less than 4 weeks after the end of treatment and presence of coccoid forms and biofilm of $H$. pylori ${ }^{8788}$ The recurrence rate in hospital-based studies varies between different countries and ethnic populations and is higher in countries with higher prevalence of $H$. pylori infection and lower 
human development index. ${ }^{8788} \mathrm{~A}$ recent systematic review and meta-analysis showed that the global annual recurrence rates after $H$. pylori eradication in clinical settings without mass eradication was $4.3 \% .^{87} \mathrm{High}$ reinfection/recrudescence has been reported in some areas such as Alaska, Vietnam and Bangladesh which may require modifications, such as treatment of the entire community at one time ${ }^{89-91}$ The annual recurrence rate in the gastric cancer prevention study in Shandong (not mass screening and eradication) was nearly $7 \%$ per person-year. ${ }^{29}$ However, the annual reinfection/recrudescence rate was only $1 \%$ in mass in the Matsu Islands, where the participation rates for screening and eradication therapy were $82 \%$ among the total population. ${ }^{40}$ This indicates that if the majority of infected subjects are treated in the community, the reinfection rate would be very low.

\section{CQ 18. Is a confirmation test for successful treatment of $\boldsymbol{H}$. pylori needed after mass eradication?}

Statement 18: Confirmation test of $H$. pylori eradication is not mandatory in mass screening, but should be performed in subsets of the population for assessment of treatment efficacy.

Agreement: agree (96\%).

Grade of recommendation: strong (67\%), weak (33\%), weak against $(0 \%)$, strong against $(0 \%)$

Evidence level: low.

\section{Comments}

A confirmation test after $H$. pylori eradication therapy is recommended for symptomatic $H$. pylori infected patients. $^{246278}$ Ideally, a confirmation test should also be offered to all asymptomatic subjects in mass screening, although this will increase the cost. Additionally, it is important to monitor the eradication rate of the regimen used in that population. For example, the eradication rate of omeprazole and amoxicillin was 62\% (703/1130) in the Shandong study, whereas the eradication rate of 10 -day bismuth quadruple therapy was $72.9 \%$ in the mass eradication programme in Linqu County, China. ${ }^{392}$ Since the effectiveness of $H$. pylori eradication for gastric cancer prevention is expected to be higher if the eradication rate is higher, it is recommended that a confirmation test should be performed at least in subsets of the population to assess treatment efficacy.

\section{POTENTIALLY ADVERSE CONSEQUENCES OF $\boldsymbol{H}$. PYLORI ERADICATION}

\section{CQ 19. Does treatment of $\boldsymbol{H}$. pylori with antibiotics increase} the antibiotic resistance rate of other bacteria?

Statement 19: As with all antibiotic treatments, H. pylori eradication may lead to an increase in antimicrobial resistance, but it should not preclude its use for gastric cancer prevention.

Agreement: agree (92\%).

Grade of recommendation: strong (58\%), weak (38\%), weak against (4\%), strong against (0\%).

Evidence level: very low.

\section{Comments}

Emergence of antibiotic resistance with the widespread use of antibiotics is one of the major concerns that limit mass screening and eradication of $H$. pylori for gastric cancer prevention. ${ }^{8}$ We identified five studies that assessed the shortterm changes of phenotypic resistance of intestinal microflora and three studies that reported the short-term changes of clarithromycin resistance $\operatorname{erm}(\mathrm{B})$ genes. $^{93-97}$ These studies collectively showed that the antibiotic resistance rates of surrogate intestinal bacteria are increased shortly after $H$. pylori eradication. ${ }^{93-99}$ The long-term phenotypic and genotypic changes of antibiotic resistance of intestinal microflora were assessed in three studies. ${ }^{93}$ The antibiotic resistance of $E$. coli was significantly increased 2 weeks after triple therapy or concomitant therapy, but not after bismuth quadruple therapy. Interestingly, the antibiotic resistance was restored to basal state at 2 months and 1 year. ${ }^{97}$ Hsu et al also showed that the increase in the abundance of $\operatorname{erm}(\mathrm{B})$ gene in faecal samples at week 8 was restored to pretreatment level by week $48 .{ }^{98}$ However, limitations of the above studies include the small sample size, susceptibility testing tested only in some surrogate bacteria and the long-term changes assessed in few studies.

However, it is not wise, and not acceptable, to preclude the use of antibiotics to treat $H$. pylori infection for prevention of gastric cancer because of this concern considering that the emergence of antibiotic resistance is multifactorial. ${ }^{100} 101$ A recent review showed that the relative contribution of misuse or overuse of antibiotics in humans and animals, contamination of the environment, suboptimal dosing of antibiotics and healthcare transmission as a driver for antimicrobial resistance are moderate to high, whereas that of mass drug administration for human health is classified as low to moderate. ${ }^{101}$ Overall, there is insufficient evidence to reach a conclusion about the effect of mass eradication therapy on antibiotic resistance in the community, and more large-scale studies are needed to investigate this subject

\section{CQ 20. Does H. pylori eradication lead to long-term disturbance of gut microbiota in adults?}

Statement 20: Short-term perturbation of faecal microbiota diversity occurs after $H$. pylori eradication, which largely recovers subsequently.

Agreement: agree (88\%).

Grade of recommendation: strong (48\%), weak (48\%), weak against (4\%), strong against (0\%).

Evidence level: low.

\section{Comments}

Recent studies have shown that the diversity of gastric microbiota is lower in $H$. pylori infected subjects than in non- $H$. pylori infected subjects. ${ }^{101} 102$ After successful eradication of H. pylori, the diversity of gastric microbiota could be restored to a level similar to that in non-H. pylori infected subjects. ${ }^{103}$ Several studies showed significant perturbation of $\alpha$-diversity and $\beta$-diversity of faecal microbiota shortly after H. pylori eradication, and the extent of perturbation was significantly greater in patients receiving concomitant therapy and bismuth quadruple therapy than in those receiving triple therapy. ${ }^{9798}$ 104-106 There was a trend towards gradual recovery of diversity at 2-3 months after completion of eradication therapy with all regimens, but the speed of recovery was faster in those receiving triple therapy. ${ }^{97}$ Relatively few studies have reported the long-term changes of faecal microbiota. ${ }^{97} 104$ Two studies showed that the diversity at 1 year was restored to pretreatment state in patients receiving triple therapy, and was largely recovered in patients receiving bismuth quadruple therapy and concomitant therapy. ${ }^{97} 104$ However, significant changes in the composition and abundance at genus level were seen 1 year after eradication therapy. ${ }^{104}$ Because the $16 \mathrm{~S}$ rRNA method was used in the majority of studies, in-depth 
sequencing using shotgun metagenomics sequencing is recommended in future studies.

\section{CQ 21. Does $H$. pylori eradication lead to new-onset gastro-oesophageal reflux disease (GORD)? Does $\boldsymbol{H}$. pylori eradication aggravate the severity of symptoms in patients with existing GORD?}

Statement 21-1: Eradication of $H$. pylori does not increase the risk of new onset GORD.

Agreement: agree (92\%).

Grade of recommendation: strong (76\%), weak (16\%), weak against (8\%), strong against (0\%).

Evidence level: high.

Statement 21-2: H. pylori eradication therapy does not increase the risk of relapse of GORD.

Agreement: agree (96\%).

Grade of recommendation: strong (72\%), weak (24\%), weak against (4\%), strong against (0\%).

Evidence level: moderate.

\section{Comments}

Gastric acid secretion of hosts following $H$. pylori infection may be unchanged, increase or decrease, depending on the pattern of gastritis (pan-gastritis, antrum-predominant gastritis or corpuspredominant gastritis). Eradication of $H$. pylori can cure gastritis, restore acid secretion and influence the severity of reflux symptoms in some patients with existing GORD. ${ }^{107}$ However, $H$. pylori eradication in populations of infected subjects, on average, does not increase the risk of developing GORD. A large-scale randomised controlled trial involving 1558 infected subjects showed that $H$. pylori eradication therapy did not influence the prevalence of reflux symptoms at 2 years. ${ }^{108}$ A meta-analysis of randomised controlled trials including only $H$. pylori-infected subjects free from GORD at baseline also showed that there was no association between eradication of $H$. pylori and the development of new cases of GORD. ${ }^{109}$ In addition, current evidence shows that $H$. pylori eradication does not increase the relapse risk of GORD. Several randomised controlled trials revealed that eradication of $H$. pylori did not increase the recurrence rates of reflux symptoms or erosive oesophagitis in infected patients with pre-existing GORD (online supplementary table 4S). ${ }^{110} 111$ Therefore, the presence of GORD should not preclude practitioner from $H$. pylori eradication therapy, although $H$. pylori infection and GORD are negatively associated at a population level. ${ }^{112}$

Currently, whether $H$. pylori eradication exaggerates reflux symptoms in patients with GORD receiving long-term acid suppression remains controversial. A randomised controlled trial from a western population showed $H$. pylori eradication did not worsen reflux disease in patients with GORD receiving long-term acid suppression. ${ }^{113}$ However, another randomised controlled trial from a Chinese population demonstrated that H. pylori eradication led to worsened control of reflux disease in patients undergoing long-term low-dose PPI therapy. ${ }^{114}$ Nonetheless, long-term use of PPI in $H$. pylori-infected subjects may lead to the development of precancerous lesions, although the findings remain contradictory. ${ }^{113} 115$ In another study, Kuipers et al showed that eradication of $H$ pylori can reduce gastric mucosal inflammation and lead to regression of corpus glandular atrophy. ${ }^{113}$ Therefore, eradication of H. pylori eradication is recommended in patients with GORD receiving long-term acid-suppression therapy.
CQ 22. Does $H$. pylori eradication lead to the increase of metabolic syndrome in adults?

Statement 22: $H$. pylori eradication may be associated with a small increase in body weight, but does not increase the risk of metabolic syndrome.

Agreement: agree (80\%).

Grade of recommendation: strong (48\%), weak (44\%), weak against (8\%), strong against (0\%).

Evidence level: low.

\section{Comments}

H. pylori has been associated with obesity, metabolic syndrome and insulin resistance in earlier observational studies. ${ }^{116117}$ Casecontrol studies showed an inverse association of H. pylori and body weight. ${ }^{117}$ Some cohort studies showed an increase of body weight after $H$. pylori eradication. ${ }^{99}$ A randomised controlled trial also showed a trivial increase in body mass index 1 year after H. pylori eradication. ${ }^{118}$ The mean body mass index increased from 27.5 to $27.8 \mathrm{~kg} / \mathrm{m}^{2}$ and 27.0 to $27.2 \mathrm{~kg} / \mathrm{m}^{2}$ in the eradication group and placebo groups, respectively. ${ }^{118}$ The increase in body weight is probably attributed to the restoration of ghrelin secretion or the relief of dyspeptic symptoms. ${ }^{119}$ Yet, the clinical significance of this trivial increase in body weight remains questionable. Some studies have shown that insulin resistance, fasting glucose, total cholesterol and triglyceride levels were reduced after H. pylori eradication. ${ }^{929799120}$ The changes in these metabolic parameters might be attributed to alterations in the gut microbiota. However, these findings remain controversial and further well-designed randomised trials are warranted to clarify the impact of $H$. pylori eradication on metabolic parameters.

CQ 23. Does H. pylori eradication increase the risk of asthma, inflammatory bowel disease and other immune-related diseases in adults?

Statement 23: $\mathrm{H}$. pylori eradication does not increase the risk of asthma, inflammatory bowel disease and other immune-related diseases in adults.

Agreement: agree (80\%).

Grade of recommendation: strong (52\%), weak (44\%), weak against (4\%), strong against (0\%).

Evidence level: very low.

\section{Comments}

Some reports demonstrated inverse association of early exposure to $H$. pylori with risk for asthma, which may be attributed to the activation of Th1 cells and inhibition of Th2 allergic response by H. pylori. ${ }^{121} 122$ Although meta-analysis suggested that patients with asthma have lower prevalence rates of $H$. pylori infection, little is known about whether eradication of $H$. pylori may increase the risk of asthma in adults. ${ }^{122}$ Systematic review and meta-analysis showed an inverse association between $H$. pylori infection and inflammatory bowel disease (IBD). ${ }^{123}$ A retrospective cohort study using health insurance showed that eradication of $H$. pylori is associated with a significantly increased risk of IBD and certain autoimmune diseases in Taiwan. ${ }^{124}$ However, other studies have shown that $H$. pylori eradication therapy is not associated with onset of IBD, and some studies even showed that eradication therapy may reduce the severity of rheumatoid arthritis and thyroid autoantibodies. ${ }^{125-127}$ More large-scale prospective studies are needed to clarify the effects of $H$. pylori eradication on the incidence of IBD and autoimmune diseases in adults. 


\section{ENDOSCOPIC SURVEILLANCE FOR GASTRIC CANCER AFTER H. PYLORI ERADICATION}

CQ 24. Who needs surveillance endoscopy after $H$. pylori eradication?

Statement 24: Subjects with advanced gastric atrophy or intestinal metaplasia should receive surveillance endoscopy to detect gastric cancer after $H$. pylori eradication.

Agreement: agree (92\%).

Grade of recommendation: strong (68\%), weak (32\%), weak against (0\%), strong against (0\%).

Evidence level: low.

\section{Comments}

Patients may remain at risk of future gastric cancer even after H. pylori eradication, especially those with preneoplastic lesions, such as atrophic gastritis, intestinal metaplasia (IM) and dysplasia. The residual risk ranges from 21 to 128 per 100000 person-years, depending on pre-existing histology and genetic factors. Risk stratification is needed to identify those with the highest risk, for endoscopic surveillance. Two Japanese studies showed that patients with severe atrophy on endoscopy (O2-O3 based on the Kimura-Takemoto Classification System) had significantly higher risk (HR 9.3-14.4) of gastric cancer after a mean follow-up of 5.6-6.4 years. ${ }^{128} 129$ Patients with intestinal metaplasia at either antrum (HR 3.6) or corpus (HR 3.7) are at higher risk of gastric cancer after $H$. pylori eradication. ${ }^{128}$ Severe atrophy on histology (operative link on gastritis assessment (OLGA) stage III-IV) also correlates with a increased risk of gastric cancer (incidence rate $/ 10^{3}$ person-years 19.7-36.5 for stage III and 41.2-63.1 for stage IV) in two Italian cohort studies. ${ }^{130131}$ The operative link on gastric intestinal metaplasia assessment (OLGIM) had a higher interobserver agreement than the OLGA classification in a cross-sectional study. ${ }^{132}$ The severity of gastric intestinal metaplasia can also be assessed by the narrow-band imaging on endoscopy. ${ }^{133}$ Lower serum pepsinogen I or pepsinogen I/II ratio before eradication therapy may be an alternative marker for risk stratification. ${ }^{134} 135$ However, it is noteworthy that gastric cancer may develop even 10 years after eradication therapy in patients who have no, or only mild, precancerous lesions at baseline. ${ }^{136}$ Further prospective studies are warranted to validate the combination of endoscopic and histological grading of gastric atrophy/intestinal metaplasia. Serum biomarkers may provide better prediction of future risk of gastric cancer after $H$. pylori eradication.

\section{CQ 25. What is the appropriate endoscopic interval for subjects who retain the gastric cancer risk?}

Statement 25: Surveillance endoscopy is suggested every 2 to 3 years for subjects with advanced gastric atrophy or intestinal metaplasia, and every 12 months after the removal of neoplasia.

Agreement: agree (92\%).

Grade of recommendation: strong (52\%), weak (44\%), weak against (4\%), strong against (0\%).

Evidence level: low.

\section{Comments}

Two cohort studies showed that more gastric cancers were detected at an early stage in patients who received surveillance endoscopy for precancerous conditions than in those not surveyed. ${ }^{137} 138$ The median time interval between the initial endoscopy and the diagnosis of cancer was 25 months (range 12 30). ${ }^{138} \mathrm{~A}$ meta-analysis showed that the incidence rates of gastric cancer in patients with atrophic gastritis and intestinal metaplasia are $1.24(95 \%$ CI 0.80 to 1.76$)$ and $3.38(95 \%$ CI 2.13 to 4.85 ) cases per 1000 person-years, respectively. ${ }^{139}$ Advanced-stage atrophy and intestinal metaplasia are defined as OLGA stage III/IV and OLGIM stage III/IV, respectively. The definition is based on the study following the "MAnagement of Precancerous conditions and lesions in the Stomach (MAPS)" guideline, which showed that $3.8 \%$ of patients with OLGIM stages III/IV and/or serum pepsinogen I/II ratio $\leq 3$ progressed to high-grade dysplasia or cancer. ${ }^{140}$ Whereas the American Gastroenterology Association (AGA) recommends that routine endoscopic surveillance should not be carried out, the 2019 MAPS-2 consensus and British Society of Gastroenterology guidelines recommend endoscopic surveillance intervals of within 6 to 12 months for patients with low- or high-grade gastric dysplasia, every 3 years for patients with precancerous lesions in advanced OLGA/OLGIM stages III/IV, and a follow-up period longer than 3 years, or even not suggested, for patients with precancerous lesion confined only to the gastric antrum as with low OLGA/ OLGIM stages. ${ }^{141-143}$ Endoscopic surveillance every 1 or 3 years for extensive precancerous conditions was also shown to be cost-effective. ${ }^{144145}$ However, the OLGIM system is relatively complex in routine clinical practice in countries with a high incidence of gastric cancer, such as Japan. Precise risk stratifications according to age, blood test with new biomarkers, history and certain new earlier pathological precancerous lesion, such as CGI/SPEM, are promising for guiding the surveillance intervals after $H$. pylori eradication to further reduce the mortality due to gastric cancer.

\section{CQ 26. What is the role of molecular markers in the risk stratification of future gastric cancer risk after $\mathrm{H}$. pylori eradication?}

Statement 26: Genetic and epigenetic markers show promise in stratifying gastric cancer risk after $H$. pylori eradication, but require further validation in prospective studies.

Agreement: agree (92\%).

Grade of recommendation: strong (52\%), weak (48\%), weak against (0\%), strong against $(0 \%)$.

Evidence level: low.

\section{Comments}

Molecular markers which mark genetic and epigenetic alterations of significance in gastric carcinogenesis may be useful in risk stratification after $H$. pylori eradication. ${ }^{146}$ DNA methylation is a key epigenetic modification, and accumulation of aberrant DNA methylation, resulting in an epigenetic field defect, has been shown to be associated with gastric cancer risk. ${ }^{147} 148$ The risk of developing metachronous gastric cancers was higher in those with higher methylation level of $m i R-124 a-3 .{ }^{148} \mathrm{~A}$ nationwide, prospective multicentre cohort study for for prediction of the risk of gastric cancer in 2000 healthy individuals after $H$. pylori eradication is in progress in Japan (UMIN000016894). Following the same protocol, this genetic approach has been proved to be effective for risk stratification after $H$. pylori eradication. ${ }^{148}$ To examine the genomic landscape of IM in gastric carcinogenesis, a prospective pre-disease high-risk cohort (Gastric Cancer Epidemiology Programme) comprising 2980 subjects with mean age $59 \pm 7$ years was enrolled, and 5 years of surveillance were completed in $2016 .{ }^{149}$ Twenty-one cases of early gastric neoplasia, defined as high-grade dysplasia, carcinoma in situ or adenocarcinoma, were detected during surveillance. Comprehensive genomic profiling, using next-generation sequencing to characterise IM biopsies, was performed, and 
three genomic alterations which showed associations with disease progression of IM to early gastric neoplasia were identified. ${ }^{149}$ Shorter telomere lengths and the presence of somatic copy-number alterations correlate with progression of intestinal metaplasia, whereas normal epigenomic patterns were associated with regression. ${ }^{148}$ A customised molecular test to identify this high-risk subset of patients with IM for targeted endoscopic surveillance is being developed, and will be validated in an international, prospective multicentre cohort study.

\section{LIMITATIONS OF THE CONSENSUS}

The limitations of the supporting evidence for each statement are shown in table 1 . First, updated incidence of gastric cancer and prevalence of $H$. pylori are lacking in many countries, which may lead to bias in the estimation of the level of global disease. Second, most of the trials reporting the effectiveness of $H$. pylori eradication on gastric cancer prevention were conducted in Eastern populations, and progression of gastric precancerous lesions rather than the development of gastric cancer was the primary outcome in several trials. Additionally, the eradication rates were lower than $80 \%$ in the majority of trials, and 5-15\% of participants in the control (placebo) group were negative for H. pylori, which may lead to bias toward the null. Although we expect higher risk reduction of gastric cancer in a younger population, the mean ages were 50 years and $>60$ years in trials conducted for primary and secondary prevention of gastric cancer, respectively. Third, the assumptions used in the costeffectiveness analysis were based on observational studies rather than randomised trials and the saving related to dyspepsia or peptic ulcer disease were rarely considered in the models. Fourth, since the risk of gastric cancer differs according to gender and ethnicity, the starting age for gastric cancer prevention might differ accordingly. Yet, few studies examined this topic. Fifth, among the 26 statements, only two are of high evidence level and most are of low evidence level. The low level of evidence might be an explanation for voting 'strong against' by some

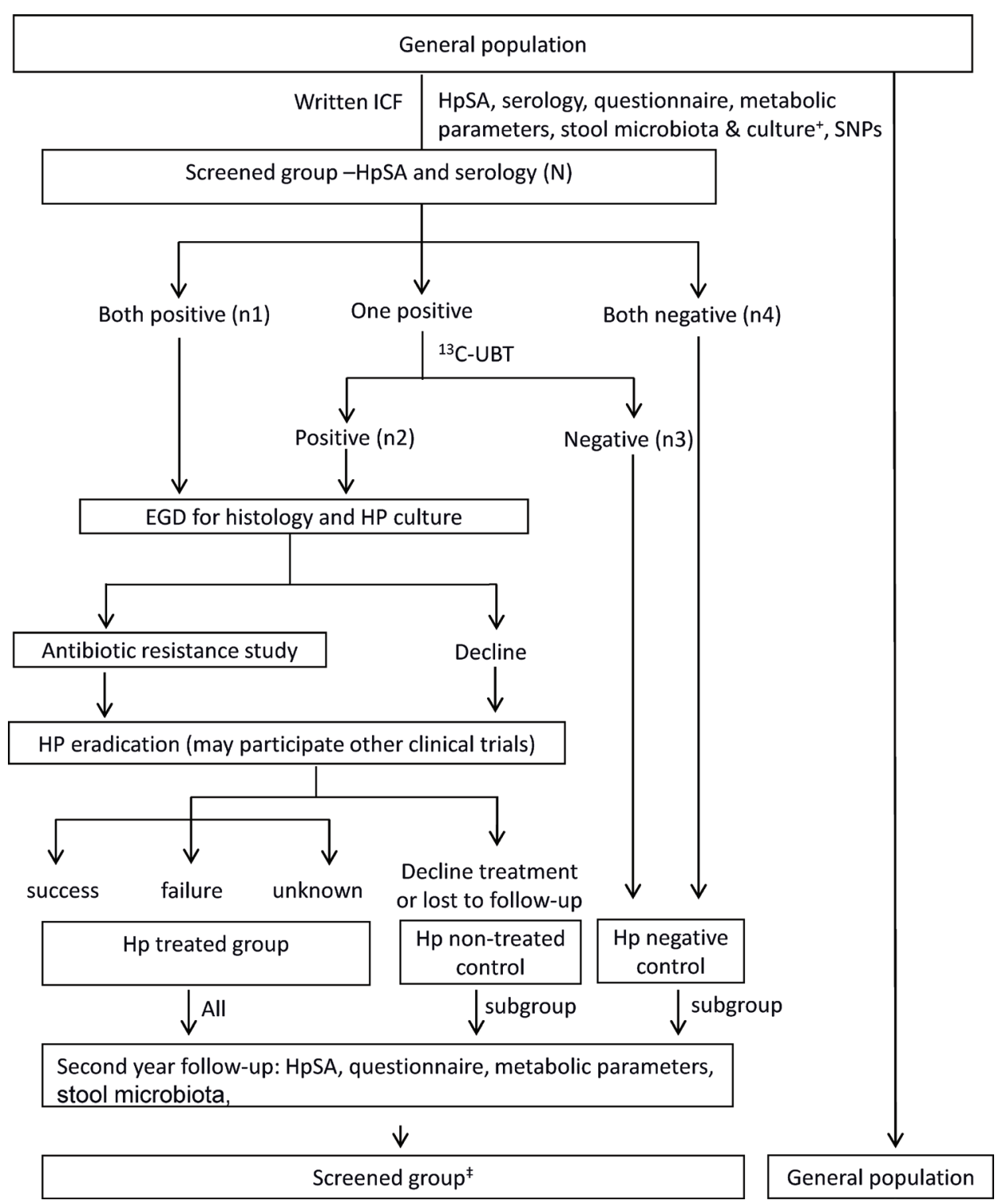

Figure 5 Study flow of the proposed collaborative cohort study. Prevalence of $H$. pylori defined as (N1 +n2/N) X 100\%; outcomes: standardised incidence rate and standardised mortality rate of gastric cancer, other $\mathrm{Gi}$ cancers in the screened group* as compared with the general population. ${ }^{13} \mathrm{C}$-UBT, ${ }^{13} \mathrm{C}$-urea breath test; EGD, esophagogastroduodenoscopy, HP, H. pylori; HpSA, H. pylori stool antigen (test); ICF, informed consent form; SNP, single nucleotide polymorphism. 
experts (4\%) for statements 2-2, 3, 4, 5,6,7,8 and 15 . Further well-designed prospective trials or cohort studies are warranted on these issues. Finally, more well-designed prospective studies or trials are warranted to assess the potential extragastroduodenal beneficial effects and long-term adverse consequences, as well as the optimal endoscopic surveillance interval following H. pylori eradication in patients with preneoplastic changes, the subset requiring long-term follow-up after successful $H$. pylori eradication. ${ }^{150}$

\section{FUTURE COLLABORATIVE RESEARCH PROPOSAL}

We designed a prospective cohort study to examine the problems raised above (figure 5). Detailed information of this proposal is available in the online supplemental materials. Researchers who are interested in this collaborative research may contact the authors. Alternatively, the protocol is open access and the researchers may conduct this trial independently. In brief, healthy adult subjects ( $\mathrm{n}=14400$ in Taiwan) will be screened for $H$. pylori infection by serology test and $H$. pylori stool antigen test (HpSA). Those with only one positive test will be confirmed by ${ }^{13} \mathrm{C}$-UBT. Endoscopy, histology and culture of $H$. pylori will be done in subgroup of subjects. Antibiotic resistance of $H$. pylori will be determined. We will link to the database of vital statistics in Taiwan to assess the short-term and long-term outcomes as follows. At the end of the cross-sectional survey, the age-standardised prevalence, the antibiotic resistance rate of H. pylori, and the age-standardised and specific incidence and mortality of gastric cancer will be reported. The standardised incidence rate and standardised mortality rate of gastric cancer and extragastric diseases in the screened group versus the general population, and the cost-effectiveness of the programme in countries with different prevalence of $H$. pylori and incidence of gastric cancer, will be the long-term endpoints.

\section{CONCLUSIONS}

Gastric cancer is attributable to H. pylori infection in nearly $90 \%$ (non-cardia) of patients, and will remain an important global health problem due to the increase in the elderly population. At an individual level, eradication of $H$. pylori reduces the risk of gastric cancer in healthy subjects and in patients with early gastric cancer following curative endoscopic resection and is recommended for all $H$. pylori infected subjects unless there are competing considerations, such as those with limited life expectancy. At a population level, the strategy of screen-and-treat for $\mathrm{H}$. pylori infection is most cost-effective in young adults in regions with a high incidence of gastric cancer and is recommended preferably before the development of atrophic gastritis and intestinal metaplasia in high-risk populations. However, population-wide screening and eradication of $H$. pylori infection should be integrated or included into the national healthcare priorities to optimise the resources. In the face of global rising resistance rates to clarithromycin and levofloxacin, we recommend proven locally effective regimens based on the principles of antibiotic stewardship. As with all antibiotic therapies, $H$. pylori eradication may lead to a short-term perturbation of faecal microbiota diversity and an increase in antimicrobial resistance, but these should not preclude its use for prevention of gastric cancer. $H$. pylori eradication does not increase the risk of new-onset GORD. There is no evidence to suggest that $H$. pylori eradication may increase the risk of metabolic syndrome, autoimmune disease and IBD in adults. Subjects with higher risk of gastric cancer, such as those with advanced gastric atrophy or intestinal metaplasia, should receive surveillance endoscopy after $H$. pylori eradication. Well-designed studies and trials are warranted to fill the knowledge gaps of the unresolved concerns.

\section{Author affiliations}

'Division of Gastroenterology and Hepatology, Department of Internal Medicine, National Taiwan University Hospital, Taipei, Taiwan

${ }^{2}$ Department of Internal Medicine, National Taiwan University College of Medicine, Taipei, Taiwan

${ }^{3}$ Department of Medicine, National Taiwan University Cancer Center, Taipei, Taiwan ${ }^{4}$ Department of Gastroenterology, Hepatology and Infectious Diseases, Otto-vonGuericke University Magdeburg, Magdeburg, Germany

${ }^{5}$ Department of Medicine II, University Hospital, LMU Munich, Munich, Germany

${ }^{6}$ Department of Medical Research, National Taiwan University Hospital, Taipei, Taiwan

${ }^{7}$ Department of Internal Medicine and Institute of Clinical Medicine, National Cheng

Kung University Hospital, College of Medicine, National Cheng Kung University,

Tainan, Taiwan

${ }^{8}$ Division of Gastroenterology, Department of Internal Medicine, Kaohsiung Medical

University Hospital, Kaohsiung, Taiwan

${ }^{9}$ Department of Medicine, Jichi Medical School, Tochigi, Japan

${ }^{10}$ Department of Internal Medicine, Tainan Hospital, Ministry of Health and Welfare, Tainan, Taiwan

"Department of Medicine, Yong Loo Lin School of Medicine, National University of Singapore, Singapore

${ }^{12}$ Division of Gastroenterology and Hepatology, Department of Internal Medicine, An Nan Hospital, China Medical University, Tainan, Taiwan

${ }^{13}$ Department of Gastroenterology and Hepatology, University of Malaya, Kuala Lumpur, Malaysia

${ }^{14}$ Department of Medicine, Faculty of Medicine, Chulalongkorn University and King

Chulalongkorn Memorial Hospital, Bangkok, Thailand

${ }^{15}$ Division of Gastroenterology and Hepatology, Department of Medicine, Nihon University School of Medicine, Tokyo, Japan

${ }^{16}$ Integrated Diagnostics and Therapeutics, National Taiwan University Hospital,

National Taiwan University College of Medicine, Taipei, Taiwan

${ }^{17}$ Institute of Biomedical Informatics, School of Medicine, National Yang-Ming

University, Taipei, Taiwan

${ }^{18}$ Division of Translational Research, Department of Medical Research, Taipei Veterans General Hospital, Taipei, Taiwan

${ }^{19}$ Department of Internal Medicine, National Taiwan University Hospital Bei-Hu Branch, Taipei, Taiwan

${ }^{20}$ Division of Gastroenterology and Hepatology, Key Laboratory of Gastroenterology and Hepatology, Shanghai Institute of Digestive Disease, Renji Hospital, School of

Medicine, Shanghai Jiao Tong University, Shanghai, China

${ }^{21}$ Oita University Faculty of Medicine, Yufu, Oita, Japan

${ }^{22}$ Department of Medicine, Michael E DeBakey VA Medical Center and Baylor College of Medicine, Houston, Texas, USA

${ }^{23}$ French National Reference Centre for Helicobacters, Bacteriology laboratory, Pellegrin Hospital, Bordeaux, \& INSERM U1053, University of Bordeaux, Bordeaux, France

${ }^{24}$ Institute of Digestive Disease, Chinese University of Hong Kong, Shatin, Hong Kong, China

${ }^{25}$ Department of Medicine and Therapeutics, The Chinese University of Hong Kong, Shatin, Hong Kong, China

${ }^{26}$ Digestive Medicine Center, China Medical University Hospital, Taichung, Taiwan

${ }^{27}$ Department of Medicine, University of New South Wales, Sydney, New South Wales, Australia

${ }^{28}$ Microbiome Research Centre, St George \& Sutherland Clinical School, University of New South Wales, Sydney, NSW, Australia

Twitter Emad M El-Omar @emadelomar

Acknowledgements The authors would like to express their special thanks to the Eighth Core Laboratory, Department of Medical Research, National Taiwan University Hospital for their technological support. DYG, M-SW, and EME-O shared the cosenior and co-corresponding authorships in this work.

Contributors Drafts of clinical questions (CQs) about each topic were prepared by J-ML and were further revised by core members (PM, Y-CL, DYG, EME-O and M-SW). Faculty members (J-ML, Y-CL, B-SS, H-CC, KG-Y, W-LC, M-JC, T-HC, C-CC, C-YW, P-IH and M-SW) were assigned to perform systematic review of CQs 1 to 4 and to prepare the statements, which were edited in the first steering committee. The revised draft statements were further edited by moderates and core members (J-ML, PM, Y-CL, B-SS, DYG, EME-O and M-SW). Faculty members (J-ML, Y-CL, B-SS, H-CC, KG-Y, W-LC, M-JC, T-HC, C-CC, C-YW, P-IH and M-SW) drafted the comments after the consensus meeting. J-ML drafted the article, which was critically revised by co-first author PM and three senior authors, DYG, M-SW and EME-O. All authors commented on drafts and approved the final version. All authors had full access to the data and participated in the decision to submit for publication. 
Funding The study was funded by the National Taiwan University Hospital (grant number: NTUH 107-P05; 109-P03), the Ministry of Science and Technology, Executive Yuan, ROC, Taiwan (grant number: TCTC 108-2321-B-002 -040 - and MOST 108-2314-B-002 -187, 108-2314-B-002 -209 -), the Ministry of Health and Welfare of Taiwan (grant number: MOHW107-TDU-B-211-123002, MOHW108CDC-C-114-112102), the "Center of Precision Medicine" from The Featured Areas Research Center Program within the framework of the Higher Education Sprout Project by the Ministry of Education (MOE) in Taiwan (grant number: NTU-107L9014-1), the Liver Disease Prevention \& Treatment Research Foundation, Taiwan, and the Gastroenterological Society of Taiwan (GEST). The GEST received funding from the Takeda Taiwan Co. Ltd (APTC-01), the Eisai Co. Ltd, the Swiss Pharmaceutical Co. (APTC-02), Ltd, the Panion \& BF Biotech Inc. (APTC-03), and the Harvester Trading Co. LTD (APTC-04). for the 10th Asian Pacific Topic Conference. DYG is supported in part by the Office of Research and Development Medical Research Service Department of Veterans Affairs, Public Health Service grant DK56338 which funds the Texas Medical Center Digestive Diseases Center. EME-O is funded by grants from the Australian Federal Government to the St George and Sutherland Medical Research Foundation. The funding source had no role in study design, data collection, analysis or interpretation, report writing or the decision to submit this paper for publication.

Competing interests J-ML reports receiving lecture fees from Takeda Pharmaceuticals (Taiwan) and Abbott Laboratories. PM reports receiving advisory and lecture fees from Bayer, Mayoly Spindler and Nordmark. DYG is a consultant for RedHill Biopharma and Phathom Pharmaceuticals regarding novel $H$. pylori therapies and has received research support for culture of Helicobacter pylori.

Patient and public involvement Patients and/or the public were not involved in the design, or conduct, or reporting, or dissemination plans of this research.

Patient consent for publication Not required.

Provenance and peer review Not commissioned; externally peer reviewed.

Data availability statement All data relevant to the study are included in the article or uploaded as supplementary information.

\section{ORCID iDs}

Jyh-Ming Liou http://orcid.org/0000-0002-7945-5408

Yi-Chia Lee http://orcid.org/0000-0002-8160-1216

Bor-Shyang Sheu http://orcid.org/0000-0002-2164-138X

Khay-Guan Yeoh http://orcid.org/0000-0002-7802-4606

Takuji Gotoda http://orcid.org/0000-0001-6904-6777

Chun-Ying Wu http://orcid.org/0000-0001-5053-1801

Hong Lu http://orcid.org/0000-0002-3127-6048

Yoshio Yamaoka http://orcid.org/0000-0002-1222-5819

Francis K L Chan http://orcid.org/0000-0001-7388-2436

Jaw-Town Lin http://orcid.org/0000-0001-9368-6141

David Y Graham http://orcid.org/0000-0002-6908-8317

Ming-Shiang Wu http://orcid.org/0000-0001-5325-3974

Emad M El-Omar http://orcid.org/0000-0002-0011-3924

\section{REFERENCES}

1 Bray F, Ferlay J, Soerjomataram I, et al. Global cancer statistics 2018: GLOBOCAN estimates of incidence and mortality worldwide for 36 cancers in 185 countries. CA Cancer J Clin 2018;68:394-424.

2 IARC Working group on the evaluation of carcinogenic risks to humans. Schistosomes, liver flukes and Helicobacter pylori. IARC Monogr Eval Carcinog Risks Hum 1994;1994:1-241.

3 Wong BC-Y, Lam SK, Wong WM, et al. Helicobacter pylori eradication to prevent gastric cancer in a high-risk region of China: a randomized controlled trial. JAMA 2004;291:187-94.

4 Choi IJ, Kook M-C, Kim Y-I, et al. Helicobacter pylori therapy for the prevention of metachronous gastric cancer. N Engl J Med 2018;378:1085-95.

5 Choi IJ, Kim CG, Lee JY, et al. Family history of gastric cancer and Helicobacter pylori treatment. N Engl J Med 2020;382:427-36.

6 Lee Y-C, Chiang T-H, Chou C-K, et al. Association between Helicobacter pylori eradication and gastric cancer incidence: a systematic review and meta-analysis. Gastroenterology 2016;150:1113-24.

7 Ford AC, Yuan Y, Moayyedi P. Helicobacter pylori eradication therapy to prevent gastric cancer: systematic review and meta-analysis. Gut 2020;69:2113-2121.

8 O'Connor A, O'Morain CA, Ford AC. Population screening and treatment of Helicobacter pylori infection. Nat Rev Gastroenterol Hepatol 2017;14:230-40.

9 Liou J-M, Lee Y-C, El-Omar EM, et al. Efficacy and long-term safety of H. pylori eradication for gastric cancer prevention. Cancers 2019;11:593.

10 Herrero R, Parsonnet J, Greenberg ER. Prevention of gastric cancer. JAMA 2014;312:1197-8.

11 Herrero R, Park JY, Forman D. The fight against gastric cancer - the IARC Working Group report. Best Pract Res Clin Gastroenterol 2014;28:1107-14.
12 Atkins $D$, Best D, Briss PA, et al. Grading quality of evidence and strength of recommendations. BMJ 2004:328:1490.

13 Global cancer Observatory (GCO). Available: https://gco.iarc.fr/

14 Hooi JKY, Lai WY, Ng WK, et al. Global prevalence of Helicobacter pylori infection: systematic review and meta-analysis. Gastroenterology 2017;153:420-9.

15 Sonnenberg A, Turner KO, Genta RM. Low prevalence of Helicobacter pylori-positive peptic ulcers in private outpatient endoscopy centers in the United States. Am J Gastroenterol 2020;115:244-50.

16 Watanabe $\mathrm{M}$, Ito $\mathrm{H}$, Hosono $\mathrm{S}$, et al. Declining trends in prevalence of Helicobacter pylori infection by birth-year in a Japanese population. Cancer Sci 2015; 106:1738-43.

17 Chen M-J, Fang Y-J, Wu M-S, et al. Application of Helicobacter pylori stool antigen test to survey the updated prevalence of Helicobacter pylori infection in Taiwan. J Gastroenterol Hepatol 2020;35:233-40.

18 Suerbaum S, Michetti P. Helicobacter pylori infection. N Engl J Med 2002;347:1175-86.

19 Tang MYL, Chung PHY, Chan HY, et al. Recent trends in the prevalence of Helicobacter pylori in symptomatic children: a 12-year retrospective study in a tertiary centre. J Pediatr Surg 2019:54:255-7.

20 Curado MP, de Oliveira MM, de Araújo Fagundes M. Prevalence of Helicobacter pylori infection in Latin America and the Caribbean populations: a systematic review and meta-analysis. Cancer Epidemiol 2019;60:141-8.

21 Bauer S, Krumbiegel P, Richter M, et al. Influence of sociodemographic factors on Helicobacter pylori prevalence variability among schoolchildren in Leipzig, Germany. A long-term follow-up study. Cent Eur J Public Health 2011;19:42-5.

22 Oliveira C, Pinheiro H, Figueiredo J, et al. Familial gastric cancer: genetic susceptibility, pathology, and implications for management. Lancet Oncol 2015;16:e60-70.

23 Yusefi AR, Bagheri Lankarani K, Bastani P, et al. Risk factors for gastric cancer: a systematic review. Asian Pac J Cancer Prev 2018:19:591-603.

24 Malfertheiner P, Megraud F, O'Morain CA, et al. Management of Helicobacter pylori infection-the Maastricht V/Florence consensus report. Gut 2017:66:6-30.

25 Naseem M, Barzi A, Brezden-Masley C, et al. Outlooks on Epstein-Barr virus associated gastric cancer. Cancer Treat Rev 2018;66:15-22.

26 Uemura N, Okamoto S, Yamamoto S, et al. Helicobacter pylori infection and the development of gastric cancer. N Engl J Med 2001;345:784-9.

27 Plummer M, Franceschi S, Vignat J, et al. Global burden of gastric cancer attributable to Helicobacter pylori. Int J Cancer 2015;136:487-90.

28 Correa Pet al. Chemoprevention of gastric dysplasia: randomized trial of antioxidant supplements and anti-Helicobacter pylori therapy. J Nat/ Cancer Inst 2000:92:1881-8

29 You W-cheng, Brown LM, Zhang L, et al. Randomized double-blind factorial trial of three treatments to reduce the prevalence of precancerous gastric lesions. J Nat/ Cancer Inst 2006:98:974-83.

30 Zhou L, Sung JJY, Lin S, et al. A five-year follow-up study on the pathological changes of gastric mucosa after H. pylori eradication. Chin Med J 2003:116:11-14.

31 Leung WK, Lin S-R, Ching JYL, et al. Factors predicting progression of gastric intestinal metaplasia: results of a randomised trial on Helicobacter pylori eradication. Gut 2004;53:1244-9.

32 Saito D, Boku N, Fujioka T, et al. Impact of H-pylori eradication on gastric cancer prevention: endoscopic results of the Japanese intervention trial (JITHP-study). A randomized multi-center trial. Gastroenterology 2005;128:A4

33 Ma J-L, Zhang L, Brown LM, et al. Fifteen-year effects of Helicobacter pylori, garlic, and vitamin treatments on gastric cancer incidence and mortality. J Nat/ Cancer Inst 2012:104:488-92.

34 Li W-Q, Zhang J-Y, Ma J-L, et al. Effects of Helicobacter pylori treatment and vitamin and garlic supplementation on gastric cancer incidence and mortality: follow-up of a randomized intervention trial. BMJ 2019;366:15016.

35 Wong BCY, Zhang L, Ma J-ling, et al. Effects of selective COX-2 inhibitor and Helicobacter pylori eradication on precancerous gastric lesions. Gut 2012;61:812-8.

36 Lee Y-C, Chen TH-H, Chiu H-M, et al. The benefit of mass eradication of Helicobacter pylori infection: a community-based study of gastric cancer prevention. Gut 2013:62:676-82

37 Chiang T-H, Chang W-J, Chen SL-S, et al. Mass eradication of Helicobacter pylori to reduce gastric cancer incidence and mortality: a long-term cohort study on Matsu Islands. Gut. In press 2020;2018:gutinl-2020-322200.

38 Fukase K, Kato M, Kikuchi S, et al. Effect of eradication of Helicobacter pylori on incidence of metachronous gastric carcinoma after endoscopic resection of early gastric cancer: an open-label, randomised controlled trial. Lancet 2008;372:392-7.

39 Choi JM, Kim SG, Choi J, et al. Effects of Helicobacter pylori eradication for metachronous gastric cancer prevention: a randomized controlled trial. Gastrointest Endosc 2018:88:475-85.

40 Fan F, Wang Z, Li B, et al. Effects of eradicating Helicobacter pylori on metachronous gastric cancer prevention: a systematic review and meta-analysis. J Eval Clin Pract 2020:26:308-15

41 Pan K-feng, Zhang L, Gerhard M, et al. A large randomised controlled intervention trial to prevent gastric cancer by eradication of Helicobacter pylori in Linqu County, China: baseline results and factors affecting the eradication. Gut 2016;65:9-18. 
42 Sugano K. Effect of Helicobacter pylori eradication on the incidence of gastric cancer: a systematic review and meta-analysis. Gastric Cancer 2019;22:435-45.

43 Graham DY, Shiotani A. The time to eradicate gastric cancer is now. Gut 2005:54:735-8.

44 Graham DY. Helicobacter pylori update: gastric cancer, reliable therapy, and possible benefits. Gastroenterology 2015;148:719-31.

45 Wu C-Y, Kuo KN, Wu M-S, et al. Early Helicobacter pylori eradication decreases risk of gastric cancer in patients with peptic ulcer disease. Gastroenterology 2009;137:e1-2.

46 Correa P. Human gastric carcinogenesis: a multistep and multifactorial process--First American Cancer Society award lecture on cancer epidemiology and prevention. Cancer Res 1992;52:6735-40.

47 Selgrad M, Bornschein J, Kandulski A, et al. Combined gastric and colorectal cancer screening —a new strategy. Int J Mol Sci 2018;19:3854.

48 Leung WK, Wong IOL, Cheung KS, et al. Effects of Helicobacter pylori treatment on incidence of gastric cancer in older individuals. Gastroenterology 2018:155:67-75

49 Areia M, Carvalho R, Cadime AT, et al. Screening for gastric cancer and surveillance of premalignant lesions: a systematic review of cost-effectiveness studies. Helicobacter 2013;18:325-37.

50 Lansdorp-Vogelaar I, Sharp L. Cost-effectiveness of screening and treating Helicobacter pylori for gastric cancer prevention. Best Pract Res Clin Gastroenterol 2013;27:933-47

51 Parsonnet J, Harris RA, Hack HM, et al. Modelling cost-effectiveness of Helicobacter pylori screening to prevent gastric cancer: a mandate for clinical trials. The Lancet 1996:348:150-4.

52 Lee Y-C, Lin J-T, Wu H-M, et al. Cost-effectiveness analysis between primary and secondary preventive strategies for gastric cancer. Cancer Epidemiol Biomarkers Prev 2007; 16:875-85.

53 Cheng H-C, Wang J-D, Chen W-Y, et al. Helicobacter pylori test-and-treat program can be cost-effective to prevent gastric cancer in Taiwanese adults: referred to the nationwide reimbursement database. Helicobacter 2015;20:114-24.

54 Chen Q, Liang X, Long X, et al. Cost-Effectiveness analysis of screen-and-treat strategy in asymptomatic Chinese for preventing Helicobacter pylori-associated diseases. Helicobacter 2019;24:e12563.

55 Shin DW, Yun YH, Choi IJ, et al. Cost-effectiveness of eradication of Helicobacter pylori in gastric cancer survivors after endoscopic resection of early gastric cancer. Helicobacter 2009; 14:536-44.

56 Weyermann M, Rothenbacher D, Brenner H. Acquisition of Helicobacter pylor infection in early childhood: independent contributions of infected mothers, fathers, and siblings. Am J Gastroenterol 2009;104:182-9.

57 Lee Y-C, Tseng P-H, Liou J-M, et al. Performance of a one-step fecal sample-based test for diagnosis of Helicobacter pylori infection in primary care and mass screening settings. J Formos Med Assoc 2014;113:899-907.

58 Lee Y-C, Lin J-T, Lee Yi-Chia. Screening and treating Helicobacter pylori infection for gastric cancer prevention on the population level. J Gastroenterol Hepatol 2017;32:1160-9

59 Best LM, Takwoingi Y, Siddique S, et al. Non-Invasive diagnostic tests for Helicobacter pylori infection. Cochrane Database Syst Rev 2018;3:CD012080.

60 Boklage SH, Mangel AW, Ramamohan V, et al. Impact of patient adherence on the cost-effectiveness of noninvasive tests for the initial diagnosis of Helicobacter pylori infection in the United States. Patient Prefer Adherence 2016;10:45-55.

61 Lee Y-C, Chiu H-M, Chiang T-H, et al. Accuracy of faecal occult blood test and Helicobacter pylori stool antigen test for detection of upper gastrointestinal lesions. BMJ Open 2013;3:e003989.

62 Chey WD, Leontiadis GI, Howden CW, et al. Acg clinical guideline: treatment of Helicobacter pylori infection. Am J Gastroenterol 2017;112:212-39.

63 Sugano K, Tack J, Kuipers EJ, et al. Kyoto global consensus report on Helicobacter pylori gastritis. Gut 2015;64:1353-67.

64 Tan MC, Graham DY. Gastric cancer risk stratification and surveillance after Helicobacter pylori eradication: 2020. Gastrointest Endosc 2019:90:457-60.

65 Liou J-M, Lin J-T, Wang H-P, et al. The optimal age threshold for screening upper endoscopy for uninvestigated dyspepsia in Taiwan, an area with a higher prevalence of gastric cancer in young adults. Gastrointest Endosc 2005:61:819-25.

66 Yaghoobi M, McNabb-Baltar J, Bijarchi R, et al. What is the quantitative risk of gastric cancer in the first-degree relatives of patients? A meta-analysis. WJG 2017:23:2435-42.

67 Bang CS, Lee JJ, Baik GH. Prediction of chronic atrophic gastritis and gastric neoplasms by serum pepsinogen assay: a systematic review and meta-analysis of diagnostic test accuracy. J Clin Med 2019;8:657.

68 Lee Y-C, Chiang T-H, Liou J-M, et al. Mass eradication of Helicobacter pylori to prevent gastric cancer: theoretical and practical considerations. Gut Liver 2016:10:12-26.

69 Hiroi S, Sugano K, Tanaka S, et al. Impact of health insurance coverage for Helicobacter pylori gastritis on the trends in eradication therapy in Japan: retrospective observational study and simulation study based on real-world data. BMJ Open 2017;7:e015855.
70 Lee Y-C, Chiang T-H, Chiu H-M, et al. Mo1637 - screening and treating Helicobacter pylori integrated with the fecal immunochemical test: a community-based randomized trial. Gastroenterology 2019;156:S-811-S-812

71 Bair M-J, Chuang S-L, Lei W-Y, et al. Planning mass eradication of Helicobacter pylori infection for Indigenous Taiwanese peoples to reduce gastric cancer. J Gastroenterol Hepatol 2020:35:609-16.

72 Wu J-Y, Lee Y-C, Graham DY. The eradication of Helicobacter pylori to prevent gastric cancer: a critical appraisal. Expert Rev Gastroenterol Hepatol 2019:13:17-24.

73 Kuo Y-T, Liou J-M, El-Omar EM, et al. Primary antibiotic resistance in Helicobacter pylori in the Asia-Pacific region: a systematic review and meta-analysis. Lancet Gastroenterol Hepatol 2017;2:707-15.

74 Savoldi A, Carrara E, Graham DY, et al. Prevalence of antibiotic resistance in Helicobacter pylori: a systematic review and meta-analysis in World Health Organization regions. Gastroenterology 2018;155:1372-82.

75 Dyar OJ, Huttner B, Schouten J, et al. What is antimicrobial stewardship? Clin Microbiol Infect 2017;23:793-8

76 Graham DY. Illusions regarding Helicobacter pylori clinical trials and treatment guidelines. Gut 2017;66:2043-6.

77 Graham DY, Tansel A. Interchangeable use of proton pump inhibitors based on relative potency. Clin Gastroenterol Hepatol 2018;16:800-8.

78 Graham DY, Lu H, Dore MP. Relative potency of proton-pump inhibitors, Helicobacter pylori therapy cure rates, and meaning of double-dose PPI. Helicobacter 2019;24:e12554.

79 Graham DY, Dore MP. Update on the use of vonoprazan: a competitive acid blocker. Gastroenterology 2018;154:462-6.

80 Sheu B-S, Wu M-S, Chiu C-T, et al. Consensus on the clinical management, screening-to-treat, and surveillance of Helicobacter pylori infection to improve gastric cancer control on a nationwide scale. Helicobacter 2017:22:e12368.

81 Suzuki S, Gotoda T, Kusano C, et al. Seven-day vonoprazan and low-dose amoxicillin dual therapy as first-line Helicobacter pylori treatment: a multicentre randomised trial in Japan. Gut 2020;69:1019-26.

82 Murakami K, Sakurai Y, Shiino M, et al. Vonoprazan, a novel potassium-competitive acid blocker, as a component of first-line and second-line triple therapy for Helicobacter pylori eradication: a phase III, randomised, double-blind study. Gut 2016:65:1439-46.

83 Liou J-M, Chen P-Y, Luo J-C, et al. Efficacies of genotypic resistance-guided vs empirical therapy for refractory Helicobacter pylori infection. Gastroenterology 2018; 155:1109-19.

84 Chen Q, Long X, Ji Y, et al. Randomised controlled trial: susceptibility-guided therapy versus empiric bismuth quadruple therapy for first-line Helicobacter pylori treatment. Aliment Pharmacol Ther 2019:49:1385-94.

85 Take S, Mizuno M, Ishiki K, et al. Reinfection rate of Helicobacter pylori after eradication treatment: a long-term prospective study in Japan. $J$ Gastroenterol 2012:47:641-6

86 Ailloud F, Didelot $X$, Woltemate $S$, et al. Within-host evolution of Helicobacter pylori shaped by niche-specific adaptation, intragastric migrations and selective sweeps. Nat Commun 2019;10:2273.

87 Hu Y, Wan J-H, Li X-Y, et al. Systematic review with meta-analysis: the global recurrence rate of Helicobacter pylori. Aliment Pharmacol Ther 2017;46:773-9.

88 Yan T-L, Hu Q-D, Zhang Q, et al. National rates of Helicobacter pylori recurrence are significantly and inversely correlated with human development index. Aliment Pharmacol Ther 2013:37:963-8.

89 Nolen LD, Vindigni SM, Parsonnet J, et al. Combating gastric cancer in Alaska native people: an expert and community symposium. Gastroenterology 2020:158:1197-201.

90 Hildebrand P, Bardhan P, Rossi L, et al. Recrudescence and reinfection with Helicobacter pylori after eradication therapy in Bangladeshi adults. Gastroenterology 2001;121:792-8

91 Wheeldon T-U, Hoang TTH, Phung DC, et al. Long-term follow-up of Helicobacter pylori eradication therapy in Vietnam: reinfection and clinical outcome. Aliment Pharmacol Ther 2005;21:1047-53.

92 Abdel-Razik A, Mousa N, Shabana W, et al. Helicobacter pylori and non-alcoholic fatty liver disease: a new enigma? Helicobacter 2018;23:e12537.

93 Sjlund M, Wreiber K, Andersson DI, et al. Long-term persistence of resistant Enterococcus species after antibiotics to eradicate Helicobacter pylori. Ann Intern Med 2003:139:483-7.

94 Jakobsson $\mathrm{H}$, Wreiber $\mathrm{K}$, Fall $\mathrm{K}$, et al. Macrolide resistance in the normal microbiota after Helicobacter pylori treatment. Scand I Infect Dis 2007:39:757-63.

95 Stark CA, Adamsson I, Edlund C, et al. Effects of omeprazole and amoxycillin on the human oral and gastrointestinal microflora in patients with Helicobacter pylori infection. J Antimicrob Chemother 1996;38:927-39.

96 Adamsson I, Nord CE, Lundquist P, et al. Comparative effects of omeprazole, amoxycillin plus metronidazole versus omeprazole, clarithromycin plus metronidazole on the oral, gastric and intestinal microflora in Helicobacter pylori-infected patients. Antimicrob Chemother 1999:44:629-40.

97 Liou J-M, Chen C-C, Chang C-M, et al. Long-term changes of gut microbiota, antibiotic resistance, and metabolic parameters after Helicobacter pylori eradication: a multicentre, open-label, randomised trial. Lancet Infect Dis 2019;19:1109-20. 
98 Hsu P-I, Pan C-Y, Kao JY, et al. Short-term and long-term impacts of Helicobacter pylori eradication with reverse hybrid therapy on the gut microbiota. J Gastroenterol Hepatol 2019;34:1968-76.

99 Jakobsson HE, Jernberg C, Andersson AF, et al. Short-term antibiotic treatment has differing long-term impacts on the human throat and gut microbiome. PLoS One 2010;5:e9836.

100 Holmes AH, Moore LSP, Sundsfjord A, et al. Understanding the mechanisms and drivers of antimicrobial resistance. The Lancet 2016:387:176-87.

101 Castro-Sánchez E, Moore LSP, Husson F, et al. What are the factors driving antimicrobial resistance? perspectives from a public event in London, England. BMC Infect Dis 2016;16:465.

102 Vasapolli R, Schütte K, Schulz C, et al. Analysis of transcriptionally active bacteria throughout the gastrointestinal tract of healthy individuals. Gastroenterology 2019; 157:1081-92

103 Guo Y, Zhang Y, Gerhard M, et al. Effect of Helicobacter pylori on gastrointestinal microbiota: a population-based study in Linqu, a high-risk area of gastric cancer. Gut 2020:69:1598-607.

104 Liou J-M, Lee Y-C, Wu M-S. Treatment of Helicobacter pylori infection and its longterm impacts on gut microbiota. J Gastroenterol Hepatol 2020;35:1107-16.

105 Chen L, Xu W, Lee A, et al. The impact of Helicobacter pylori infection, eradication therapy and probiotic supplementation on gut microenvironment homeostasis: an open-label, randomized clinical trial. EBioMedicine 2018;35:87-96.

106 Gotoda T, Takano C, Kusano C, et al. Gut microbiome can be restored without adverse events after Helicobacter pylori eradication therapy in teenagers. Helicobacter 2018;23:e12541.

107 Malfertheiner P, Peitz U. The interplay between Helicobacter pylori, gastrooesophageal reflux disease, and intestinal metaplasia. Gut 2005;54 Suppl 1:i13-20.

108 Harvey RF, Lane JA, Murray LJ, et al. Randomised controlled trial of effects of Helicobacter pylori infection and its eradication on heartburn and gastrooesophageal reflux: Bristol Helicobacter project. BMJ 2004;328:1417.

109 Yaghoobi M, Farrokhyar F, Yuan Y, et al. Is there an increased risk of GERD after Helicobacter pylori eradication?: a meta-analysis. Am J Gastroenterol 2010;105:1007-13.

110 Schwizer W, Menne D, Schütze K, et al. The effect of Helicobacter pylori infection and eradication in patients with gastro-oesophageal reflux disease: a parallel-group, double-blind, placebo-controlled multicentre study. United European Gastroenterol J 2013;1:226-35.

111 Wu JCY, Chan FKL, Wong SKH, et al. Effect of Helicobacter pylori eradication on oesophageal acid exposure in patients with reflux oesophagitis. Aliment Pharmacol Ther 2002;16:545-52.

112 O'Connor HJ. Review article: Helicobacter pylori and gastro-oesophageal reflux disease-clinical implications and management. Aliment Pharmacol Ther 1999:13:117-27.

113 Kuipers EJ, Nelis GF, Klinkenberg-Knol EC, et al. Cure of Helicobacter pylori infection in patients with reflux oesophagitis treated with long term omeprazole reverses gastritis without exacerbation of reflux disease: results of a randomised controlled trial. Gut 2004;53:12-20.

114 Wu JCY, Chan FKL, Ching JYL, et al. Effect of Helicobacter pylori eradication on treatment of gastro-oesophageal reflux disease: a double blind, placebo controlled, randomised trial. Gut 2004:53:174-9.

115 Kuipers EJ, Lundell L, Klinkenberg-Knol EC, et al. Atrophic gastritis and Helicobacter pylori infection in patients with reflux esophagitis treated with omeprazole or fundoplication. N Engl J Med 1996:334:1018-22.

116 Tsay F-W, Hsu P-I. H. pylori infection and extra-gastroduodenal diseases. J Biomed Sci 2018;25:65

117 Wu M-S, Lee W-J, Wang H-H, et al. A case-control study of association of Helicobacter pylori infection with morbid obesity in Taiwan. Arch Intern Med 2005; 165:1552-5.

118 Lane JA, Murray LJ, Harvey IM, et al. Randomised clinical trial: Helicobacter pylori eradication is associated with a significantly increased body mass index in a placebo-controlled study. Aliment Pharmacol Ther 2011;33:922-9.

119 Osawa H, Nakazato M, Date Y, et al. Impaired production of gastric ghrelin in chronic gastritis associated with Helicobacter pylori. J Clin Endocrinol Metab 2005:90:10-16.

120 Cheng K-P, Yang Y-J, Hung H-C, et al. Helicobacter pylori eradication improves glycemic control in type 2 diabetes patients with asymptomatic active Helicobacter pylori infection. J Diabetes Investig 2019;10:1092-101.

121 Amedei A, Codolo G, Del Prete G, et al. The effect of Helicobacter pylori on asthma and allergy. J Asthma Allergy 2010;3:139-47.

122 Zhou X, Wu J, Zhang G. Association between Helicobacter pylori and asthma: a meta-analysis. Eur J Gastroenterol Hepatol 2013;25:460-8

123 Castaño-Rodríguez N, Kaakoush NO, Lee WS, et al. Dual role of Helicobacter and Campylobacter species in IBD: a systematic review and meta-analysis. Gut 2017:66:235-49

124 Lin K-D, Chiu G-F, Waljee AK, et al. Effects of anti-Helicobacter pylori therapy on incidence of autoimmune diseases, including inflammatory bowel diseases. Clin Gastroenterol Hepatol 2019:17:1991-9.
125 Hou Y, Sun W, Zhang C, et al. Meta-analysis of the correlation between Helicobacter pylori infection and autoimmune thyroid diseases. Oncotarget 2017;8:115691-700.

126 Rosania R, Von Arnim U, Link A, et al. Helicobacter pylori eradication therapy is not associated with the onset of inflammatory bowel diseases. A case-control study. J Gastrointestin Liver Dis 2018;27:119-25.

127 Zentilin P, Seriolo B, Dulbecco P, et al. Eradication of Helicobacter pylori may reduce disease severity in rheumatoid arthritis. Aliment Pharmacol Ther 2002;16:1291-9.

128 Shichijo S, Hirata Y, Niikura R, et al. Histologic intestinal metaplasia and endoscopic atrophy are predictors of gastric cancer development after Helicobacter pylori eradication. Gastrointest Endosc 2016:84:618-24.

129 Abnet CC, Zheng W, Ye W, et al. Plasma pepsinogens, antibodies against Helicobacter pylori, and risk of gastric cancer in the Shanghai women's health study cohort. Br J Cancer 2011;104:1511-6.

130 Rugge M, Genta RM, Fassan M, et al. OLGA gastritis staging for the prediction of gastric cancer risk: a long-term follow-up study of 7436 patients. Am J Gastroenterol 2018:113:1621-8.

131 Rugge M, Meggio A, Pravadelli C, et al. Gastritis staging in the endoscopic follow-up for the secondary prevention of gastric cancer: a 5-year prospective study of 1755 patients. Gut 2019;68:11-17

132 Capelle LG, de Vries AC, Haringsma J, et al. The staging of gastritis with the OLGA system by using intestinal metaplasia as an accurate alternative for atrophic gastritis. Gastrointest Endosc 2010:71:1150-8.

133 Esposito G, Pimentel-Nunes P, Angeletti S, et al. Endoscopic grading of gastric intestinal metaplasia (EGGIM): a multicenter validation study. Endoscopy 2019;51:515-21.

134 Chiang T-H, Chiu SY-H, Chen SL-S, et al. Serum pepsinogen as a predictor for gastric cancer death: a 16-year community-based cohort study. J Clin Gastroenterol 2019:53:e186-93.

135 Chiang TH, Maeda M, Yamada H, et al. Risk stratification for gastric cancer after Helicobacter pylorieradication: a population-based study on Matsu islands. $J$ Gastroenterol Hepatol 2020;8.

136 Take S, Mizuno M, Ishiki K, et al. Seventeen-year effects of eradicating Helicobacter pylori on the prevention of gastric cancer in patients with peptic ulcer; a prospective cohort study. J Gastroentero/ 2015;50:638-44.

137 Whiting JL, Sigurdsson A, Rowlands DC, et al. The long term results of endoscopic surveillance of premalignant gastric lesions. Gut 2002;50:378-81.

138 Rokkas T, Filipe MI, Sladen GE. Detection of an increased incidence of early gastric cancer in patients with intestinal metaplasia type III who are closely followed up. Gut 1991;32:1110-3.

139 Akbari M, Tabrizi R, Kardeh S, et al. Gastric cancer in patients with gastric atrophy and intestinal metaplasia: a systematic review and meta-analysis. PLoS One 2019;14:e0219865

140 den Hollander WJ, Holster IL, den Hoed CM, et al. Surveillance of premalignant gastric lesions: a multicentre prospective cohort study from low incidence regions. Gut 2019;68:585-93.

141 Gupta S, Li D, El Serag HB, et al. AGA clinical practice guidelines on management of gastric intestinal metaplasia. Gastroenterology 2020;158:693-702.

142 Pimentel-Nunes P, Libânio D, Marcos-Pinto R, et al. Management of epithelial precancerous conditions and lesions in the stomach (maps II): European Society of Gastrointestinal Endoscopy (ESGE), European Helicobacter and Microbiota Study Group (EHMSG), European Society of Pathology (ESP), and Sociedade Portuguesa de Endoscopia Digestiva (SPED) guideline update 2019. Endoscopy 2019:51:365-88.

143 Banks M, Graham D, Jansen M, et al. British Society of Gastroenterology guidelines on the diagnosis and management of patients at risk of gastric adenocarcinoma. Gut 2019;68:1545-75.

144 Hassan C, Zullo A, Di Giulio E, et al. Cost-effectiveness of endoscopic surveillance for gastric intestinal metaplasia. Helicobacter 2010;15:221-6.

145 Areia M, Dinis-Ribeiro M, Rocha Gonçalves F. Cost-utility analysis of endoscopic surveillance of patients with gastric premalignant conditions. Helicobacter 2014:19:425-36.

146 Maeda M, Yamashita S, Shimazu T, et al. Novel epigenetic markers for gastric cancer risk stratification in individuals after Helicobacter pylori eradication. Gastric Cancer 2018;21:745-55.

147 Asada K, Nakajima T, Shimazu T, et al. Demonstration of the usefulness of epigenetic cancer risk prediction by a multicentre prospective cohort study. Gut 2015;64:388-96.

148 Ando T, Yoshida T, Enomoto S, et al. DNA methylation of microRNA genes in gastric mucosae of gastric cancer patients: its possible involvement in the formation of epigenetic field defect. Int J Cancer 2009;124:2367-74.

149 Huang KK, Ramnarayanan K, Zhu F, et al. Genomic and epigenomic profiling of highrisk intestinal metaplasia reveals molecular determinants of progression to gastric cancer. Cancer Cell 2018:33:137-50.

150 Malfertheiner P. Helicobacter pylori treatment for gastric cancer prevention. N Eng/ J Med 2018:378:1154-6. 